\title{
Finite Curvature of Arc Length Measure Implies Rectifiability: A New Proof \\ XAVIER TOLSA
}

\begin{abstract}
If $E \subset \mathbb{C}$ is a set with finite length and finite curvature, then $E$ is rectifiable. This fact, proved by David and Léger in 1999 , is one of the basic ingredients for the proof of Vitushkin's conjecture. In this paper we give another different proof of this result.
\end{abstract}

\section{INTRODUCTION}

Given three pairwise different points $x, y, z \in \mathbb{C}$, their Menger curvature is

$$
c(x, y, z)=\frac{1}{R(x, y, z)},
$$

where $R(x, y, z)$ is the radius of the circumference passing through $x, y, z$ (with $R(x, y, z)=\infty, c(x, y, z)=0$ if $x, y, z$ lie on a same line). If two among these points coincide, we let $c(x, y, z)=0$. If $\mu$ is a Radon measure on $\mathbb{C}$, we define the curvature of $\mu$ as

$$
c^{2}(\mu)=\iiint c(x, y, z)^{2} d \mu(x) d \mu(y) d \mu(z) .
$$

The notion of curvature of a measure was introduced by Mel'nikov [16] when he was studying a discrete version of analytic capacity, and it is one of the ideas which is responsible of the big recent advances in connection with analytic capacity.

Let $\mathcal{H}^{1}$ denote the 1-dimensional Hausdorff measure (also called arc length measure). Recall that a set $E \subset \mathbb{C}$ is said to be rectifiable if there exists a countable 
family of rectifiable curves $\Gamma_{i}$ such that $\mathcal{H}^{1}\left(E \backslash \bigcup_{i} \Gamma_{i}\right)=0$. On the other hand, $E$ is called purely unrectifiable if it intersects any rectifiable curve at most in a set of zero length.

The following result was proved by David and Léger [10].

Theorem 1.1. Let $E \subset \mathbb{C}$ be a compact set with $\mathcal{H}^{1}(E)<\infty$ such that $c^{2}\left(\mathcal{H}_{\mid E}^{1}\right)<$ $\infty$. Then $E$ is rectifiable.

The proof of this result in [10] uses ideas which go back to the Jones' development of quantitative rectifiability [8] and to the geometric corona decompositions used by David and Semmes in their pioneering study of uniform rectifiability [3]. See also [11] for another recent result closely related to Theorem 1.1 which also uses techniques of a similar nature.

In this paper we will show another proof of Theorem 1.1. Our proof is very different from the one in [10] and, perhaps, simpler. It is based on the use of a sharp inequality relating analitic capacity and curvature, and also on the characterization of purely unrectifiable sets in terms of lower 1-dimensional densities by Besicovitch (see (1.4) below). However, unfortunately our arguments don't yield the quantitative version of Theorem 1.1 stated in [10, Proposition 1.1], and they don't extend to compact sets $E$ in $\mathbb{R}^{n}, n>2$, unlike in [10]. Nevertheless, we still think that our proof has its own interest.

Theorem 1.1 is one of the basic ingredients of the proof of Vitushkin's conjecture by G. David [2]. To state David's result in detail, we need to introduce additional notation and terminology.

A compact set $E \subset \mathbb{C}$ is removable for bounded analytic functions if for any open set $\Omega$ containing $E$, every bounded function analytic on $\Omega \backslash E$ has an analytic extension to $\Omega$. The analytic capacity of $E$ is

$$
\gamma(E)=\sup \left|f^{\prime}(\infty)\right|,
$$

where the supremum is taken over all analytic functions $f: \mathbb{C} \backslash E \rightarrow \mathbb{C}$ with $|f| \leq 1$ on $\mathbb{C} \backslash E$, and $f^{\prime}(\infty)=\lim _{z \rightarrow \infty} z(f(z)-f(\infty))$.

In [1], Ahlfors showed that $E$ is removable for bounded analytic functions if and only if $\gamma(E)=0$.

Painlevé's problem consists in characterizing removable singularities for bounded analytic functions in a metric/geometric way. By Ahlfors' result this is equivalent to describing compact sets with positive analytic capacity in metric/geometric terms.

Guy David [2] proved in 1998 the following result, previously conjectured by Vitushkin.

Theorem $\boldsymbol{A}$ (Vitushkin's conjecture). Let $E \subset \mathbb{C}$ be compact with $\mathcal{H}^{1}(E)<\infty$. Then, $\gamma(E)=0$ if and only if $E$ is purely unrectifiable.

To be precise, let us remark that the "if" part of the theorem is not due to David. In fact, it follows from Calderón's theorem on the $L^{2}$ boundedness of the Cauchy transform on Lipschitz graphs with small Lipschitz constant. The "only 
if" part of the theorem, which is more difficult, is the one proved by David. The proof of this implication consists of two basic steps. In the first one, given a set $E \subset \mathbb{C}$ with $\mathcal{H}^{1}(E)<\infty$ and $\gamma(E)>0$, one has to show that there exists some compact subset $F \subset E$ with $\mathcal{H}^{1}(F)>0$ such that the Cauchy transform (see the definition in next section) is bounded on $L^{2}\left(\mathcal{H}_{\mid F}^{1}\right)$. This is accomplished by means of a suitable $T(b)$ type theorem. By the relationship between curvature and the $L^{2}$ norm of the Cauchy transform (see (2.3)), one infers that $c^{2}\left(\mathcal{H}_{\mid F}^{1}\right)<\infty$. The second step of the proof of the "only if" part of Theorem A is precisely Theorem 1.1 .

Theorem A is not valid without the assumption that $E$ has finite length (see [12] and [9]). However, the notion of curvature of measures still plays a key role in the understanding of analytic capacity, as the following result, proved in [21], shows.

Theorem $B$. For any compact $E \subset \mathbb{C}$ we have

$$
\gamma(E) \approx \sup \mu(E),
$$

where the supremum is taken over all Borel measures $\mu$ supported on $E$ such that $\mu(B(x, r)) \leq r$ for all $x \in E, r>0$ and $c^{2}(\mu) \leq \mu(E)$.

The notation $A \approx B$ in the theorem means that there exists an absolute constant $C>0$ such that $C^{-1} A \leq B \leq C A$.

Previously to [21], Mark Melnikov obtained in [16] the following estimate involving analytic capacity and curvature:

$$
\gamma(E) \geq C_{1}^{-1} \sup _{\mu} \frac{\mu(E)^{2}}{\mu(E)+c^{2}(\mu)},
$$

where $C_{1}>0$ is an absolute constant and the supremum is taken over all Radon measures $\mu$ supported on $E$ such that $\mu(B(x, r)) \leq r$ for all $x \in \mathbb{C}, r>0$. Later on, in [20] it was proved that (1.3) also holds (with a constant different from $C_{1}$ ) if one replaces $\gamma$ by $\gamma_{+}\left(\right.$see $(2.2)$ for the definition of $\left.\gamma_{+}\right)$. Moreover, it was also shown that one can even take the supremum in (1.3) over the (larger) collection of Radon measures $\mu$ supported on $E$ such that $\Theta^{*}(x, \mu) \leq 1$, where $\Theta^{*}(x, \mu)$ stands for the upper 1-dimensional density of $\mu$ at $x$ :

$$
\Theta^{*}(x, \mu)=\limsup _{r \rightarrow 0} \frac{\mu(B(x, r))}{2 r} .
$$

Recall that the corresponding lower density is defined by

$$
\Theta_{*}(x, \mu)=\liminf _{r \rightarrow 0} \frac{\mu(B(x, r))}{2 r} .
$$


When $\mu$ is the 1 -dimensional Hausdorff measure $\mathcal{H}^{1}$ over a $\mathcal{H}^{1}$-measurable set $E \subset \mathbb{C}$, we write $\Theta^{*}(x, E)=\Theta^{*}\left(x, \mathcal{H}_{\mid E}^{1}\right)$, and analogously with $\Theta_{*}$.

In this paper we will prove an inequality similar to (1.3). This will be an essential tool in our proof of Theorem 1.1. The precise result is the following.

Theorem 1.2. Let $E \subset \mathbb{C}$ be compact. We have

$$
\gamma(E) \geq \sup _{\mu} \frac{\mu(E)^{2}}{\frac{4 \pi}{3} \mu(E)+\frac{1}{6 \pi} c^{2}(\mu)},
$$

where the supremum is taken over all Radon measures $\mu$ supported on $E$ such that

$$
\Theta^{*}(x, \mu)<\infty \quad \text { and } \quad \Theta_{*}(x, \mu) \leq 1
$$

at $\mu$-a.e. $x \in E$.

Let us remark that (1.5) becomes an identity when $E$ is a circumference of radius $R$ : if we take $\mu$ equal to the arc length measure on $E$, then $\mu(E)=2 \pi R$ and

$$
c^{2}(\mu)=\frac{1}{R^{2}}(2 \pi R)^{3}=8 \pi^{3} R .
$$

Thus,

$$
\frac{\mu(E)^{2}}{\frac{4 \pi}{3} \mu(E)+\frac{1}{6 \pi} c^{2}(\mu)}=\frac{4 \pi^{2} R^{2}}{\frac{8 \pi^{2}}{3} R+\frac{8 \pi^{3}}{6 \pi} R}=R=\gamma(E) .
$$

On the other hand, if $E$ is a segment of length $L$, and we choose $\mu$ as the arc length on $E$ again, we have

$$
\frac{\mu(E)^{2}}{\frac{4 \pi}{3} \mu(E)+\frac{1}{6 \pi} c^{2}(\mu)}=\frac{L^{2}}{\frac{4 \pi}{3} L+0}=\frac{3}{4 \pi} L<\frac{1}{4} L=\gamma(E) .
$$

So the inequality is not sharp for a segment. However, the number $3 /(4 \pi)$ is not very far from $\frac{1}{4}$, at least for our purposes. This fact will be essential in our proof of Theorem 1.1.

\section{Preliminaries}

As usual, in the paper the letter ' $C$ ' stands for an absolute constant which may change its value at different occurrences. On the other hand, the constants with subscripts, such as $C_{1}$, retain their value at different occurrences. By a square we mean a closed square with sides parallel to the axes. 
A Radon measure $\mu$ on $\mathbb{R}^{d}$ has linear growth if there exists some constant $C_{0}$ such that

$$
\mu(B(x, r)) \leq C_{0} r \quad \text { for all } x \in \mathbb{C}, r>0 .
$$

Given a finite complex Radon measure $v$ on $\mathbb{C}$, the Cauchy transform of $v$ is defined by

$$
c \nu(z)=\int \frac{1}{\xi-z} d v(\xi) \text {. }
$$

Although the integral above is absolutely convergent a.e. with respect to the Lebesgue measure, it does not make sense, in general, for $z \in \operatorname{supp}(v)$. This is the reason why one considers the truncated Cauchy transform of $v$, which is defined as

$$
C_{\varepsilon} v(z)=\int_{|\xi-z|>\varepsilon} \frac{1}{\xi-z} d v(\xi),
$$

for any $\varepsilon>0$ and $z \in \mathbb{C}$. This integral now makes sense for all $z \in \mathbb{C}$. The principal value of the Cauchy transform of $v$ at $z$ is, if it exists,

$$
\text { p.v. } C \mathcal{v}(z):=\lim _{\varepsilon \rightarrow 0} C_{\varepsilon} \nu(z) \text {. }
$$

The capacity $\gamma_{+}$of a compact set $E \subset \mathbb{C}$ is

$$
\gamma_{+}(E):=\sup \left\{\mu(E) \mid \operatorname{supp}(\mu) \subset E,\|C \mu\|_{L^{\infty}(\mathbb{C})} \leq 1\right\} .
$$

That is, $\gamma_{+}$is defined as $\gamma$ in (1.2) with the additional constraint that $f$ should coincide with $C \mu$, where $\mu$ is some positive Radon measure supported on $E$ (observe that $(C \mu)^{\prime}(\infty)=-\mu(\mathbb{C})$ for any Radon measure $\left.\mu\right)$. To be precise, there is another slight difference: in (1.2) we asked $\|f\|_{L^{\infty}(\mathbb{C} \backslash E)} \leq 1$, while in (2.2), $\|f\|_{L^{\infty}(\mathbb{C})} \leq 1$ (for $f=C \mu$ ). Trivially, we have $\gamma_{+}(E) \leq \gamma(E)$. On the other hand, in [21] it has been shown that there exists an absolute constant $C$ such that $\gamma(E) \leq C \gamma_{+}(E)$ for any compact set $E \subset \mathbb{C}$. Thus, $\gamma(E) \approx \gamma_{+}(E)$.

Recall the definition of curvature of a measure in (1.1). This notion is connected to the Cauchy transform by the following result, proved by Melnikov and Verdera [17].

Proposition 2.1. Let $\mu$ be a Radon measure on $\mathbb{C}$ with linear growth. We have

$$
\left\|C_{\varepsilon} \mu\right\|_{L^{2}(\mu)}^{2}=\frac{1}{6} c_{\varepsilon}^{2}(\mu)+O(\mu(\mathbb{C})),
$$

where $|O(\mu(\mathbb{C}))| \leq C \mu(\mathbb{C})$.

In this proposition, $c_{\varepsilon}^{2}(\mu)$ stands for the $\varepsilon$-truncated version of $c^{2}(\mu)$ (defined as in the right hand side of (1.1), but with the triple integral over $\{x, y, z \in \mathbb{C}$ : $|x-y|,|y-z|,|x-z|>\varepsilon\})$. 
The identity (2.3) is remarkable because it relates an analytic notion (the Cauchy transform of a measure) with a metric-geometric one (curvature). For recent connected results, look at [22].

Given a point $x \in \mathbb{C}$, we denote

$$
c_{\mu}^{2}(x)=\iint c(x, y, z)^{2} d \mu(y) d \mu(z) .
$$

So, $c_{\mu}^{2}(x)$ is a pointwise version of $c^{2}(\mu)$, in a sense.

For open problems in connection with curvature of measures, we recommend the reader to have a look at the nice survey [14].

\section{Proof of Theorem 1.1 Using Theorem 1.2}

We will need the following well known lemma about curvature of measures, whose proof we show for completeness.

Lemma 3.1. Let $\mu$ be some positive finite Radon measure without atoms on $\mathbb{C}$. If $\mathrm{c}^{2}(\mu)<\infty$, then, for $\mu$-almost all $x \in \mathbb{C}$,

$$
\lim _{r \rightarrow 0} \frac{c^{2}\left(\mu_{\mid B(x, r)}\right)}{\mu(B(x, r))}=0
$$

Proof. For each $m \geq 1$, let

$$
A_{m}=\left\{x \in \mathbb{C} \mid \limsup _{r \rightarrow 0} c^{2}\left(\mu_{\mid B(x, r)}\right)[\mu(B(x, r))]^{-1}>\frac{1}{m}\right\} .
$$

For $r>0$, we denote

$$
c_{r}^{2}(\mu)=\iiint_{|x-y| \leq r} c(x, y, z)^{2} d \mu(x) d \mu(y) d \mu(z) .
$$

Notice that $\lim _{r \rightarrow 0} c_{r}^{2}(\mu)=0$, because $c^{2}(\mu)<\infty$ and $\mu$ has no atoms.

Given any $r>0$ and $m \geq 1$, for each $x \in A_{m}$ there exists some ball $B(x, s)$ with $s<r / 2$ such that $\mu(B(x, s)) \leq m c^{2}\left(\mu_{\mid B(x, s)}\right)$. With this type of balls, we consider a Besicovitch covering of $A_{m}$. That is, $A_{m} \subset \bigcup_{i} B\left(x_{i}, s_{i}\right)$, with $\sum_{i} \chi_{B\left(x_{i}, s_{i}\right)} \leq C$. Then,

$$
\mu\left(A_{m}\right) \leq \sum_{i} \mu\left(B\left(x_{i}, s_{i}\right)\right) \leq m \sum_{i} c^{2}\left(\mu_{\mid B\left(x_{i}, s_{i}\right)}\right) \leq C m c_{r}^{2}(\mu),
$$

which tends to 0 as $r \rightarrow 0$. Thus $\mu\left(A_{m}\right)=0$ for each $m$.

We will use the following elementary lemma too. 
Lemma 3.2. Let $E \subset \mathbb{C}$ be compact and $\mu$ supported on $E$. Suppose that there exists some $x_{0} \in E$ such that $c_{\mu}^{2}\left(x_{0}\right) \leq \delta$. Then there exists a line $L$ passing through $x_{0}$ such that for any $\varepsilon>0$

$$
\mu\{y \in E \mid \operatorname{dist}(y, L)>\varepsilon \operatorname{diam}(E)\} \leq \frac{\delta^{1 / 2} \operatorname{diam}(E)}{2 \varepsilon} .
$$

Proof. Since $c_{\mu}^{2}\left(x_{0}\right) \leq \delta$, there exists some $z_{0} \in E$ such that

$$
\int c\left(x_{0}, z_{0}, y\right)^{2} d \mu(y) \leq \frac{\delta}{\mu(E)} .
$$

Let $L$ be the line through $x_{0}$ and $z_{0}$. Recall that

$$
c\left(x_{0}, z_{0}, y\right)=\frac{2 \operatorname{dist}(y, L)}{\left|x_{0}-y\right|\left|z_{0}-y\right|} .
$$

Then we have

$$
\begin{aligned}
\mu\{y & \in E \mid \operatorname{dist}(y, L)>\varepsilon \operatorname{diam}(E)\} \\
& \leq \int \frac{\operatorname{dist}(y, L)}{\varepsilon \operatorname{diam}(E)} d \mu(y) \\
& \leq \frac{\operatorname{diam}(E)}{2 \varepsilon} \int \frac{2 \operatorname{dist}(y, L)}{\left|x_{0}-y\right|\left|z_{0}-y\right|} d \mu(y) \\
& \leq \frac{\operatorname{diam}(E) \mu(E)^{1 / 2}}{2 \varepsilon}\left(\int c\left(x_{0}, z_{0}, y\right)^{2} d \mu(y)\right)^{1 / 2} \\
& \leq \frac{\operatorname{diam}(E) \delta^{1 / 2}}{2 \varepsilon} .
\end{aligned}
$$

Notice that the preceding lemma implies that if $\mu(E) \approx \operatorname{diam}(E)$ and $\delta \ll \varepsilon^{2}$, then most of the $\mu$-mass of $E$ will be contained in an $(\varepsilon \operatorname{diam}(E))$-neighborhood of some line $L$.

Let us recall the definition of upper convex 1-dimensional density of a set $E \subset \mathbb{C}$ at $x$ :

$$
\Theta_{C}^{*}(x, E)=\lim _{r \rightarrow 0}\left(\sup \frac{\mathcal{H}^{1}(E \cap U)}{\operatorname{diam}(U)}\right),
$$

where the supremum is over all convex sets $U$ with $x \in U$ and $0<\operatorname{diam}(U) \leq r$. Recall also that if $E \subset \mathbb{C}$ is $\mathcal{H}^{1}$-measurable and $\mathcal{H}^{1}(E)<\infty$, then

$$
\Theta_{\mathcal{C}}^{*}(x, E)=1 \quad \text { at } \mathcal{H}^{1} \text {-a.e. } x \in E .
$$

See [4, Theorem 2.3], for example. Another important result due to Besicovitch fundamental for our arguments is that if, moreover, $E$ is purely unrectifiable, then

$$
\Theta_{*}(x, E) \leq \frac{3}{4} \quad \text { at } \mathcal{H}^{1} \text {-a.e. } x \in E .
$$


See [4, Theorem 3.23].

Proof of Theorem 1.1. To prove the theorem it is enough to show that if $E \subset$ $\mathbb{C}$ has finite length and it is purely unrectifiable, then $c^{2}\left(\mathcal{H}_{\mid E}^{1}\right)=\infty$.

We suppose that $c^{2}\left(\mathcal{H}_{\mid E}^{1}\right)<\infty$ and we argue by contradiction. Let $\varepsilon_{0}>0$ be such that for any segment $L_{r}$ with length $r$,

$$
\gamma\left(U_{\varepsilon_{0} r}\left(L_{r}\right)\right) \leq\left(1+\frac{1}{1000}\right) \gamma\left(L_{r}\right),
$$

where $U_{\varepsilon_{0} r}\left(L_{r}\right)$ stands for the $\varepsilon_{0} r$-neighborhood of $L_{r}$. Take $x_{0} \in E$ with $\Theta_{\mathcal{C}}^{*}\left(x_{0}, E\right)=1$ and so that

$$
\lim _{r \rightarrow 0} \frac{c^{2}\left(\mathcal{H}_{\mid E \cap B\left(x_{0}, r\right)}^{1}\right)}{\mathcal{H}^{1}\left(E \cap B\left(x_{0}, r\right)\right)}=0 .
$$

Given a small constant $\varepsilon>0$ to be fixed below (with $\varepsilon<\frac{1}{10}$, say), let $\delta>0$ be such that

$$
\mathcal{H}^{1}\left(E \cap B\left(x_{0}, r\right)\right) \leq 3 r \quad \text { for } 0<r \leq \delta,
$$

and

$$
c^{2}\left(\mathcal{H}_{\mid E \cap B\left(x_{0}, r\right)}^{1}\right) \leq \varepsilon^{3} \mathcal{H}^{1}\left(E \cap B\left(x_{0}, r\right)\right) \quad \text { for } 0<r \leq \delta .
$$

Let $F$ be a convex set containing $x_{0}$ with $\operatorname{diam}(F) \leq \delta / 2$ satisfying

$$
\frac{\mathcal{H}^{1}(F \cap E)}{\operatorname{diam}(F)} \geq 1-\varepsilon
$$

We denote $d=\operatorname{diam}(F)$. The estimate (3.1) implies that there exists some $y_{0} \in$ $E \cap B\left(x_{0}, r\right)$ such that $c_{\mathcal{H}_{\mid E \cap B\left(x_{0}, r\right)}^{1}}^{2}\left(y_{0}\right) \leq \varepsilon^{3}$. Thus, by Lemma 3.2, if $\varepsilon$ has been chosen small enough, there exists some line $\ell$ such that a big portion of $\mu$-mass of $E \cap F$ is contained in a neighborhood $U_{\varepsilon_{0} d}(\ell \cap F)$. More precisely, since $\mathcal{H}^{1}(F \cap$ $E) \approx d$, there exists some line $\ell$ such that

$$
\mathcal{H}^{1}\left(U_{\varepsilon_{0} d}(\ell \cap F) \cap E\right) \geq(1-2 \varepsilon) d .
$$

We set

$$
F^{\prime}:=U_{\varepsilon_{0} d}(\ell \cap F) \cap E .
$$

Let us denote by $L_{d}$ a segment with length $d$ containing $\ell \cap F$ (which may coincide with $\ell \cap F)$. Notice that, by the choice of $\varepsilon_{0}$, we have

$$
\gamma\left(F^{\prime}\right) \leq \gamma\left(U_{\varepsilon_{0} d}\left(L_{d}\right)\right) \leq\left(1+\frac{1}{1000}\right) \gamma\left(L_{d}\right)=\left(1+\frac{1}{1000}\right) \frac{d}{4},
$$


where we used the fact that the analytic capacity of a segment equals one quarter of its length (see Chapter VIII of [5], for example).

Now we take the measure

$$
\mu=\frac{4}{3} \mathcal{H}_{\mid F^{\prime}}^{1}
$$

Since $F^{\prime}$ is purely unrectifiable, $\Theta_{*}\left(x, F^{\prime}\right) \leq \frac{3}{4}$ at $\mathcal{H}^{1}$-a.e. $x \in F^{\prime}$. Thus,

$$
\Theta_{*}(x, \mu) \leq 1 \quad \text { at } \mu \text {-a.e. } x \in F^{\prime} .
$$

Therefore, by Theorem 1.2 we get

$$
\gamma\left(F^{\prime}\right) \geq \frac{\mu\left(F^{\prime}\right)^{2}}{\frac{4 \pi}{3} \mu\left(F^{\prime}\right)+\frac{1}{6 \pi} c^{2}(\mu)} .
$$

Observe that, by (3.1) and (3.2),

$$
c^{2}(\mu) \leq \frac{4^{3}}{3^{3}} \varepsilon^{3} \mathcal{H}^{1}\left(E \cap B\left(x_{0}, d\right)\right) \leq C \varepsilon^{3} \mu\left(F^{\prime}\right) .
$$

Thus, by (3.3),

$$
\gamma\left(F^{\prime}\right) \geq \mu\left(F^{\prime}\right) \frac{1}{4 \pi / 3+C \varepsilon^{3}} \geq d(1-2 \varepsilon) \frac{4 / 3}{4 \pi / 3+C \varepsilon^{3}} .
$$

Clearly, this estimate contradicts (3.4) if $\varepsilon$ has been chosen small enough.

\section{THEOREM 1.2 FOR Finite UNIONS OF PAIRWISE DisJOINT SEGMENTS}

To prove Theorem 1.2 we will show first that the inequality (1.5) holds when $E$ is a finite disjoint union of segments and $\mu$ is the arc length measure on $E$. In the next section we will prove (1.5) in full generality by approximation by segments.

Lemma 4.1. Let $E \subset \mathbb{C}$ be a finite union of disjoint compact segments. We have

$$
\gamma(E) \geq \frac{\mathcal{H}^{1}(E)^{2}}{\frac{4 \pi}{3} \mathcal{H}^{1}(E)+\frac{1}{6 \pi} c^{2}\left(\mathcal{H}_{\mid E}^{1}\right)} .
$$

To prove the lemma we will need to use the identity $\gamma=\left(\gamma_{2}\right)^{2}$. Recall that if $E$ is a finite union of pairwise disjoint analytic Jordan curves, then

$$
\gamma_{2}(E)=\sup \left|f^{\prime}(\infty)\right|,
$$


where the supremum is taken over all functions $f \in H^{2}(\Omega)$ such that $f(\infty)=0$, $\|f\|_{H^{2}(\Omega)} \leq 1$, where $\Omega$ stands for the unbounded component of $\mathbb{C}_{\infty} \backslash E$ and $H^{2}(\Omega)$ is the Hardy space of those functions which are analytic in $\Omega$ and

$$
\|f\|_{H^{2}(\Omega)}:=\limsup _{\varepsilon \rightarrow 0}\left(\frac{1}{2 \pi} \int_{\partial\left(\Omega \backslash U_{\varepsilon}(E)\right)}|f(z)|^{2} d \mathcal{H}^{1}(z)\right)^{1 / 2}<\infty .
$$

Let us remark that there are other equivalent (and perhaps more natural) definitions of $H^{2}(\Omega)$.

A well known result of Garabedian [6], [7, p. 22], asserts that $\gamma(E)=\gamma_{2}(E)^{2}$. It is not difficult to see that this result also holds if $E$ is made up of a finite disjoint union of analytic Jordan arcs, in particular if $E$ is a finite disjoint union of compact segments. The definition of $H^{2}(\Omega)$ is in this case the same as above.

Proof of Lemma 4.1. Let $\left\{L_{i}\right\}_{i \in I}$ be the finite collection of compact pairwise disjoint segments such that $E=\bigcup_{i \in I} L_{i}$. Consider the function $f(z):=$ $C\left(\mathcal{H}_{\mid E}^{1}\right)(z)$. This is an analytic function vanishing at $\infty$, and it is easily seen that $f \in H^{2}(\Omega)$, where $\Omega=\mathbb{C}_{\infty} \backslash E$. Thus,

$$
\gamma(E)=\gamma_{2}(E)^{2} \geq \frac{\left|f^{\prime}(\infty)\right|^{2}}{\|f\|_{H^{2}(\Omega)}^{2}} .
$$

Since $f^{\prime}(\infty)=-\mathcal{H}^{1}(E)$, the lemma follows if we show that

$$
\left\|C\left(\mathcal{H}_{\mid E}^{1}\right)\right\|_{H^{2}(\Omega)}^{2}=\frac{4 \pi}{3} \mathcal{H}^{1}(E)+\frac{1}{6 \pi} c^{2}\left(\mathcal{H}_{\mid E}^{1}\right) .
$$

To prove this identity we will show that

$$
\left\|C\left(\mathcal{H}_{\mid E}^{1}\right)\right\|_{H^{2}(\Omega)}^{2}=\pi \mathcal{H}^{1}(E)+\frac{1}{\pi} \| \text { p.v. } C\left(\mathcal{H}_{\mid E}^{1}\right) \|_{L^{2}\left(\mathcal{H}_{\mid E}^{1}\right)}^{2}
$$

and

$$
\| \text { p.v. } C\left(\mathcal{H}_{\mid E}^{1}\right) \|_{L^{2}\left(\mathcal{H}_{\mid E}^{1}\right)}^{2}=\frac{\pi^{2}}{3} \mathcal{H}^{1}(E)+\frac{1}{6} c^{2}\left(\mathcal{H}_{\mid E}^{1}\right)
$$

Let us remark that it is easy to see that the principal value p.v. $C\left(\mathcal{H}_{\mid E}^{1}\right)(z)$ exists for any $z \in E$ which is not the end point of any segment $L_{i}$. Clearly, equations (4.3) and (4.4) imply (4.2).

The identity (4.3) follows from Plemelj's formulae. Indeed, given $z \in E$ not equal to any end point of any segment $L_{i}$, let us denote by $C^{+}\left(\mathcal{H}_{\mid E}^{1}\right)(z)$ and $\mathcal{C}^{-}\left(\mathcal{H}_{\mid E}^{1}\right)(z)$ the two boundary values of $C\left(\mathcal{H}_{\mid E}^{1}\right)$ at $z$ (each boundary value corresponds to one of the two sides of the segment $L_{i}$ which contains $z$ ). Let us 
denote by $t^{+}(z)$ the unitary tangent vector ${ }^{1}$ at $z$. Then, Plemelj's formulae tell us that

$$
\left\{\begin{array}{l}
C^{+}\left(\mathcal{H}_{\mid E}^{1}\right)(z)=\text { p.v. } C\left(\mathcal{H}_{\mid E}^{1}\right)(z)+i \pi \overline{t^{+}(z)} \\
C^{-}\left(\mathcal{H}_{\mid E}^{1}\right)(z)=\text { p.v. } C\left(\mathcal{H}_{\mid E}^{1}\right)(z)-i \pi \overline{t^{+}(z)}
\end{array}\right.
$$

Then we have

$$
\begin{aligned}
2 \pi \| C\left(\mathcal{H}_{\mid E}^{1}\right) & \|_{H^{2}(\Omega)}^{2}= \\
& =\left\|C^{+}\left(\mathcal{H}_{\mid E}^{1}\right)\right\|_{L^{2}\left(\mathcal{H}_{\mid E}^{1}\right)}^{2}+\left\|C^{-}\left(\mathcal{H}_{\mid E}^{1}\right)\right\|_{L^{2}\left(\mathcal{H}_{\mid E}^{1}\right)}^{2} \\
& =\| \text { p.v. } C\left(\mathcal{H}_{\mid E}^{1}\right)+i \pi \overline{t^{+}}\left\|_{L^{2}\left(\mathcal{H}_{\mid E}^{1}\right)}^{2}+\right\| \text { p.v. } C\left(\mathcal{H}_{\mid E}^{1}\right)-i \pi \overline{t^{+}} \|_{L^{2}\left(\mathcal{H}_{\mid E}^{1}\right)}^{2} \\
& =2 \| \text { p.v. } C\left(\mathcal{H}_{\mid E}^{1}\right)\left\|_{L^{2}\left(\mathcal{H}_{\mid E}^{1}\right)}^{2}+2\right\| i \pi \bar{t}^{+} \|_{L^{2}\left(\mathcal{H}_{\mid E}^{1}\right)}^{2} \\
& =2 \| \text { p.v. } C\left(\mathcal{H}_{\mid E}^{1}\right) \|_{L^{2}\left(\mathcal{H}_{\mid E}^{1}\right)}^{2}+2 \pi^{2} \mathcal{H}^{1}(E),
\end{aligned}
$$

and so (4.3) follows.

Let us consider now equation (4.4). The identity proved by Melnikov and Verdera [17] (for the particular case we are interested in) yields

$$
\begin{aligned}
& \left\|\mathcal{C}_{\varepsilon}\left(\mathcal{H}_{\mid E}^{1}\right)\right\|_{L^{2}\left(\mathcal{H}_{\mid E}^{1}\right)}^{2}= \\
& =\frac{1}{6} \iiint_{\substack{|x-y|>\varepsilon \\
|x-z|>\varepsilon \\
|y-z|>\varepsilon}} c(x, y, z)^{2} d \mathcal{H}_{\mid E}^{1}(x) d \mathcal{H}_{\mid E}^{1}(y) d \mathcal{H}_{\mid E}^{1}(z) \\
& \quad+\iiint_{\substack{|x-y| \leq \varepsilon \\
|x-z|>\varepsilon \\
|y-z|>\varepsilon}} \frac{1}{(z-x) \overline{(z-y)}} d \mathcal{H}_{\mid E}^{1}(x) d \mathcal{H}_{\mid E}^{1}(y) d \mathcal{H}_{\mid E}^{1}(z) .
\end{aligned}
$$

It is straightforward to check that $\left\|C_{\varepsilon}\left(\mathcal{H}_{\mid E}^{1}\right)\right\|_{L^{2}\left(\mathcal{H}_{\mid E}^{1}\right)} \rightarrow \|$ p.v. $C\left(\mathcal{H}_{\mid E}^{1}\right) \|_{L^{2}\left(\mathcal{H}_{\mid E}^{1}\right)}$ as $\varepsilon \rightarrow 0$. Also it is clear that

$$
\iiint_{\substack{|x-y|>\varepsilon \\|x-z|>\varepsilon \\|y-z|>\varepsilon}} c(x, y, z)^{2} d \mathcal{H}_{\mid E}^{1}(x) d \mathcal{H}_{\mid E}^{1}(y) d \mathcal{H}_{\mid E}^{1}(z) \rightarrow c^{2}\left(\mathcal{H}_{\mid E}^{1}\right) \quad \text { as } \varepsilon \rightarrow 0 .
$$

Let us turn our attention to the last integral in (4.5). Recall that in [17] it has been shown that if $\mathcal{H}_{\mid E}^{1}$ has linear growth, then this term is bounded by $C_{2} \mathcal{H}^{1}(E)$ for all $\varepsilon>0$, with $C_{2}$ depending only on the linear growth constant. However, we are interested in the behaviour of this integral as $\varepsilon \rightarrow 0$. We claim that it converges to $\pi^{2} \mathcal{H}^{1}(E) / 3$. Clearly, (4.4) follows from this fact and the identity (4.5), letting $\varepsilon \rightarrow 0$.

We prove our claim in a separate lemma.

\footnotetext{
${ }^{1}$ The orientation of $t^{+}(z)$ must be chosen properly for Plemelj's formulae. However, the orientation of $t^{+}(z)$ is not important for our calculations, because they do not depend on the sign of $t^{+}(z)$.
} 
Lemma 4.2. Let $E \subset \mathbb{C}$ be a finite union of pairwise disjoint compact segments. We have

$$
\lim _{\varepsilon \rightarrow 0} \iiint_{\substack{|x-y|<\varepsilon \\|x-z|>\varepsilon \\|y-z|>\varepsilon}} \frac{1}{(z-x) \overline{(z-y)}} d \mathcal{H}_{\mid E}^{1}(x) d \mathcal{H}_{\mid E}^{1}(y) d \mathcal{H}_{\mid E}^{1}(z)=\frac{\pi^{2}}{3} \mathcal{H}^{1}(E) .
$$

The proof of the lemma is a rather easy exercise. However, for the reader's convenience we will show the detailed arguments.

Proof. We will see below that if $x \in E$ is not the end point of any of the segments which forms $E$, then

$$
\lim _{\varepsilon \rightarrow 0} \iint_{\substack{|x-y|<\varepsilon \\|x-z|>\varepsilon \\ y-z \mid>\varepsilon}} \frac{1}{(z-x) \overline{(z-y)}} d \mathcal{H}_{\mid E}^{1}(y) d \mathcal{H}_{\mid E}^{1}(z)=\frac{\pi^{2}}{3} .
$$

The lemma follows from this fact and the dominated convergence theorem. Indeed, it is easily seen that if $|x-y| \leq \varepsilon,|x-z|>\varepsilon$ and $|y-z|>\varepsilon$, then

$$
\left|\frac{1}{(z-x) \overline{(z-y)}}\right| \leq \frac{C}{|z-x|^{2}} \text {. }
$$

As a consequence, since there exists some constant $C_{E}$ (depending on $E$ ) such that

$$
\mathcal{H}^{1}(B(x, r) \cap E) \leq C_{E} r \quad \text { for all } x, r,
$$

using Lemma 5.1 below, we get

$$
\begin{aligned}
& \left|\iint_{\substack{|x-y| \leq \varepsilon \\
|x-z|>\varepsilon \\
y-z \mid>\varepsilon}} \frac{1}{(z-x) \overline{(z-y)}} d \mathcal{H}_{\mid E}^{1}(y) d \mathcal{H}_{\mid E}^{1}(z)\right| \\
& \quad \leq \iint_{|x-y| \leq \varepsilon} \frac{C}{|x-z|>\varepsilon} d \mathcal{H}_{\mid E}^{1}(y) d \mathcal{H}_{\mid E}^{1}(z) \\
& \quad \lesssim \int_{|x-y| \leq \varepsilon} \frac{C_{E}}{\varepsilon} d \mathcal{H}_{\mid E}^{1}(y) \leq C_{E}^{2} .
\end{aligned}
$$

Thus the dominated convergence theorem can be applied.

It remains to prove (4.6). Let us denote by $I_{\varepsilon}$ the double integral in (4.6). We split $I_{\varepsilon}$ as follows:

$$
I_{\varepsilon}=\iint_{\substack{|x-y| \leq \varepsilon \\ \varepsilon<|x-z| \leq \varepsilon^{1 / 4} \\|y-z|>\varepsilon}} \cdots+\iint_{\substack{|x-y| \leq \varepsilon \\|x-z|>\varepsilon^{1 / 4} \\|y-z|>\varepsilon}} \cdots=: I_{\varepsilon}^{1}+I_{\varepsilon}^{2}
$$


It is easy to see that $I_{\varepsilon}^{2} \rightarrow 0$ as $\varepsilon \rightarrow 0$. Indeed, for $(y, z)$ in the domain of integration of $I_{\varepsilon}^{2}$ we have $|y-z| \geq|x-z|-|x-y| \geq \varepsilon^{1 / 4}-\varepsilon$. Thus, by (4.7),

$$
\begin{aligned}
\left|I_{\varepsilon}^{2}\right| & \leq \int_{|x-y| \leq \varepsilon} \frac{\mathcal{H}^{1}(E)}{\varepsilon^{1 / 4}\left(\varepsilon^{1 / 4}-\varepsilon\right)} d \mathcal{H}_{\mid E}^{1}(y) \\
& \leq C_{E} \mathcal{H}^{1}(E) \frac{\varepsilon}{\varepsilon^{1 / 4}\left(\varepsilon^{1 / 4}-\varepsilon\right)} \rightarrow 0 \quad \text { as } \varepsilon \rightarrow 0 .
\end{aligned}
$$

Let us consider the integral $I_{\varepsilon}^{1}$ now. Let $\left\{L_{i}\right\}_{i \in I}$ be the finite collection of compact pairwise disjoint segments such that $E=\bigcup_{i \in I} L_{i}$. We denote by $L_{x}=[a, b]$ the segment which contains $x$. Recall that $x$ is not any end point of $L_{x}$. Take $\varepsilon>0$ small enough so that

$$
\varepsilon^{1 / 4}<\operatorname{dist}(x, a), \operatorname{dist}(x, b), \min _{i \in I: L_{i} \neq L_{x}} \operatorname{dist}\left(L_{x}, L_{i}\right) .
$$

Then, if $(y, z)$ is in the domain of integration of $I_{\varepsilon}^{1}$, it turns out that $y, z$ are in $L_{x}$. Moreover, since the term $1 /[(z-x) \overline{(z-y)}]$ is invariant by translations and rotations of $(x, y, z)$, we may assume that $x=0$ and that $L_{x} \subset \mathbb{R}$. Thus

$$
I_{\varepsilon}^{1}=\iint_{\substack{|y| \leq \varepsilon \\ \varepsilon<|z| \leq \varepsilon^{1 / 4} \\|y-z|>\varepsilon}} \frac{1}{z(z-y)} d y d z,
$$

where $d y$ and $d z$ denote the usual integration with respect to Lebesgue measure in $\mathbb{R}$. On the other hand, by symmetry it is easy to check that

$$
I_{\varepsilon}^{1}=2 \iint_{\substack{0<y \leq \varepsilon \\ \varepsilon<|z| \leq \varepsilon^{1 / 4} \\|y-z|>\varepsilon}} \frac{1}{z(z-y)} d y d z .
$$

Thus

$$
I_{\varepsilon}^{1}=2 \int_{0}^{\varepsilon}\left(\int_{-\varepsilon^{1 / 4}}^{-\varepsilon} \frac{1}{z(z-y)} d z+\int_{y+\varepsilon}^{\varepsilon^{1 / 4}} \frac{1}{z(z-y)} d z\right) d y .
$$

Taking into account that a primitive of $1 /(z(z-y))$ (with respect to $z$ ) is

$$
\frac{1}{y} \log \left|1-\frac{y}{z}\right|
$$

it follows that

$$
I_{\varepsilon}^{1}=2 \int_{0}^{\varepsilon}\left[\frac{2}{y} \log \left|1+\frac{y}{\varepsilon}\right|+\frac{1}{y} \log \left|1-\frac{y}{\varepsilon^{1 / 4}}\right|-\frac{1}{y} \log \left|1+\frac{y}{\varepsilon^{1 / 4}}\right|\right] d y .
$$


If we split the integral into two parts and we change variables, we get

$$
I_{\varepsilon}^{1}=4 \int_{0}^{1} \frac{1}{t} \log |1+t| d t+2 \int_{0}^{\varepsilon^{3 / 4}} \frac{1}{t}(\log |1-t|-\log |1+t|) d t .
$$

It is well known that

$$
\int_{0}^{1} \frac{1}{t} \log |1+t| d t=\frac{\pi^{2}}{12}
$$

On the other hand, the last integral in (4.8) tends to 0 as $\varepsilon \rightarrow 0$ because the function inside the integral is bounded in $\left(0, \frac{1}{2}\right]$. Thus $I_{\varepsilon}^{1} \rightarrow \pi^{2} / 3$ as $\varepsilon \rightarrow 0$, and the lemma follows.

\section{Proof of Theorem 1.2 by Approximation by Segments}

5.1. Additional notation and auxiliary lemmas. If $A \subset \mathbb{C}$ is $\mu$-measurable and $x, y \in \mathbb{C}$, we write

$$
c_{\mu}^{2}(x, y, A)=\int_{A} c(x, y, z)^{2} d \mu(z), \quad x, y \in \mathbb{C},
$$

and, if $A, B, C \subset \mathbb{C}$ are $\mu$-measurable,

$$
c_{\mu}^{2}(x, A, B)=\int_{A} \int_{B} c(x, y, z)^{2} d \mu(y) d \mu(z), \quad x \in \mathbb{C},
$$

and

$$
c_{\mu}^{2}(A, B, C)=\int_{A} \int_{B} \int_{C} c(x, y, z)^{2} d \mu(x) d \mu(y) d \mu(z) .
$$

Given two lines $\ell$ and $m, \Varangle(\ell, m)$ stands for the angle between $\ell$ and $m$ (it doesn't matter which one of the two possible angles because we will always deal with its sinus). Also, given $x, y, z \in \mathbb{C}$, we set $\Varangle(x, y, z):=\Varangle\left(\ell_{x, y}, \ell_{y, z}\right)$, where $\ell_{x, y}$ is the line through $x, y$, and analogously with $\ell_{y, z}$. Recall that for $x$, $y, z \in \mathbb{C}$ we have

$$
c(x, y, z)=\frac{2 \operatorname{dist}\left(x, \ell_{y, z}\right)}{|x-y||x-z|}=\frac{2 \sin \Varangle(x, y, z)}{|x-z|} .
$$

The following lemma will be used very often below (and has already been used).

Lemma 5.1. If $\mu$ is a Borel measure in $\mathbb{C}$ such that $\mu(B(x, r)) \leq M r$ for all $x \in \mathbb{C}, r>0$, then for all $x_{0} \in \mathbb{C}$ and $R>0$,

$$
\int_{\left|y-x_{0}\right|>R} \frac{1}{\left|y-x_{0}\right|^{2}} d \mu(y) \lesssim \frac{M}{R} .
$$


This estimate can be proved by splitting the domain of integration into annuli, for example.

We also recall the following result, whose proof follows by standard arguments (see [19, Lemma 2.4], for example).

Lemma 5.2. Let $x, y, z \in \mathbb{C}$ be three pairwise different points, and let $x^{\prime} \in \mathbb{C}$ be such that $|x-y| \leq C_{3}\left|x^{\prime}-y\right|$. Then,

$$
\left|c(x, y, z)-c\left(x^{\prime}, y, z\right)\right| \leq\left(4+2 C_{3}\right) \frac{\left|x-x^{\prime}\right|}{|x-y||x-z|} .
$$

The following estimate can be understood as a kind of interpolation property for curvature.

Lemma 5.3. Let $x, y, z \in \mathbb{C}$ be three pairwise different points, and let $x_{1}, x_{2}$ be two points such that $x, x_{1}, x_{2}$ are collinear and $\left|x-x_{1}\right| \leq C_{4}\left|x_{1}-x_{2}\right|$. Suppose that for $j=1,2$ we have $\left|x_{j}-y\right| \leq C_{5}|x-y|$ and $\left|x_{j}-z\right| \leq C_{5}|x-z|$. Then,

$$
c(x, y, z) \leq\left(C_{4}+1\right) C_{5}^{2}\left(c\left(x_{1}, y, z\right)+c\left(x_{2}, y, z\right)\right) .
$$

Proof. By elementary geometry, using the fact that $x, x_{1}, x_{2}$ are collinear, it is easy to see that

$$
\operatorname{dist}\left(x, L_{y, z}\right) \leq\left(C_{4}+1\right)\left[\operatorname{dist}\left(x_{1}, L_{y, z}\right)+\operatorname{dist}\left(x_{2}, L_{y, z}\right)\right] .
$$

Thus, by (5.1),

$$
\begin{aligned}
c(x, y, z) & \leq\left(C_{4}+1\right)\left(\frac{2 \operatorname{dist}\left(x_{1}, L_{y, z}\right)}{|x-y||x-z|}+\frac{2 \operatorname{dist}\left(x_{2}, L_{y, z}\right)}{|x-y||x-z|}\right) \\
& \leq\left(C_{4}+1\right) C_{5}^{2}\left(\frac{2 \operatorname{dist}\left(x_{1}, L_{y, z}\right)}{\left|x_{1}-y\right|\left|x_{1}-z\right|}+\frac{2 \operatorname{dist}\left(x_{2}, L_{y, z}\right)}{\left|x_{2}-y\right|\left|x_{2}-z\right|}\right) .
\end{aligned}
$$

5.2. The approximation lemma and the proof of Theorem 1.2. Theorem 1.2 follows easily from Lemma 4.1 and the next result.

Lemma 5.4. Let $\mu$ be a Radon measure supported on a compact set $E \subset \mathbb{C}$ such that $c^{2}(\mu)<\infty$ and

$$
\Theta^{*}(x, \mu)<\infty \text { and } \Theta_{*}(x, \mu) \leq 1
$$

at $\mu$-a.e. $x \in E$. For any $\varepsilon>0$, there exists a finite union of compact disjoint segments $F:=\bigcup_{i} L_{i}$ such that
(a) $F \subset U_{\varepsilon}(E)$.
(b) $\mu(E)-\varepsilon \leq \mathcal{H}^{1}(F) \leq \mu(E)$.
(c) $c^{2}\left(\mathcal{H}_{\mid F}^{1}\right) \leq c^{2}(\mu)+\varepsilon$. 
This lemma contains the most delicate part of the proof of Theorem 1.2. The lemma would be easier to prove if instead of (c) we asked for an estimate such as $c^{2}\left(\mathcal{H}_{\mid F}^{1}\right) \leq C\left(c^{2}(\mu)+\mu(E)\right)$, for some $C>1$. However, this would not suffice to prove Theorem 1.2.

To construct the set $F$ in the lemma, we will approximate $\mu$ by segments (i.e., by the arc length measure on segments). The hypothesis on the lower density of $\mu$ ensures that there is enough room to place the segments suitably. The difficult part of the proof will be the estimate of the curvature $c^{2}\left(\mathcal{H}_{\mid F}^{1}\right)$ in (c). To this end, we will need to choose the orientation of the segments in $F$ carefully.

Proof of Theorem 1.2 using Lemmas 4.1 and 5.4. Take $\varepsilon=1 / n$ in Lemma 5.4, and let $F_{n}$ be the finite union of segments satisfying the properties (a), (b) and (c) of the lemma. By Lemma 4.1 we have

$$
\begin{aligned}
\gamma\left(\overline{U_{1 / n}(E)}\right) & \geq \gamma\left(F_{n}\right) \geq \frac{\mathcal{H}^{1}\left(F_{n}\right)^{2}}{\frac{4 \pi}{3} \mathcal{H}^{1}\left(F_{n}\right)+\frac{1}{6 \pi} c^{2}\left(\mathcal{H}_{\mid F_{n}}^{1}\right)} \\
& \geq \frac{(\mu(E)-1 / n)^{2}}{\frac{4 \pi}{3} \mu(E)+\frac{1}{6 \pi}\left(c^{2}(\mu)+1 / n\right)} .
\end{aligned}
$$

If we let $n \rightarrow \infty$ in this inequality, Theorem 1.2 follows, since $\gamma\left(\overline{U_{1 / n}(E)}\right) \rightarrow \gamma(E)$ as $n \rightarrow \infty$, because of the regularity property of analytic capacity (see [5, Chapter VIII], for example).

5.3. Construction of the segments. We may assume that there exists some big constant $M$ such that $\mu(B(x, r)) \leq M r$ for all $x, r$. This follows easily from the following fact: since $\Theta *(x, \mu)<\infty \mu$-a.e., if we denote

$$
E_{n}=\{x \in E \mid \mu(B(x, r)) \leq n r \text { for all } r>0\},
$$

then $\mu\left(E \backslash \bigcup_{n} E_{n}\right)=0$. As a consequence, for $n$ big enough we have $\mu\left(E \backslash E_{n}\right) \leq$ $\varepsilon / 10$. Then we replace $\mu$ by $\mu_{\mid E_{n}}$ (notice that $\Theta_{*}\left(\mu_{\mid E_{n}}, x\right) \leq \Theta_{*}(\mu, x) \leq 1$ and $c^{2}\left(\mu_{\mid E_{n}}\right) \leq c^{2}(\mu)$, and moreover, $\mu_{\mid E_{n}}$ has linear growth, etc.).

Fix a small positive constant $\varepsilon$, with $\varepsilon<\min \left(\frac{1}{1000}, M^{-10}\right)$ say. By the condition on the lower density of $\mu$, the fact that $c^{2}(\mu)<\infty$, and Lemma 3.1, for $\mu$-a.e. $x \in E$ there exists some radius $\tilde{R}_{x}$ with $0<\tilde{R}_{x}<\varepsilon$ (which may be chosen arbitrarily small) such that

$$
\begin{gathered}
\mu\left(B\left(x, \tilde{R}_{x}\right)\right) \leq\left(2+\varepsilon^{10}\right) \tilde{R}_{x}, \\
c_{\mu \mid B(x, r)}^{2}(x) \leq \varepsilon^{150} \quad \text { if } 0<r \leq \varepsilon^{-10} \tilde{R}_{x},
\end{gathered}
$$

and moreover

$$
c^{2}\left(\mu_{\mid B(x, r)}\right) \leq \varepsilon^{150} \mu(B(x, r)) \quad \text { if } 0<r \leq \varepsilon^{-10} \tilde{R}_{x} .
$$


A key point for the arguments below is that the constant $\varepsilon^{150}$ which appears in the conditions (5.3) and (5.4) concerning curvature is very small, much smaller than other constants, such as the density constant $\theta_{0}$ that will be defined in next paragraph.

Let us distinguish two cases now. If $\mu\left(B\left(x, \tilde{R}_{x}\right)\right) \leq \theta_{0} \tilde{R}_{x}:=\varepsilon^{10} \tilde{R}_{x}$, we set $R_{x}=\varepsilon^{6} \tilde{R}_{x}$. Otherwise, we choose $R_{x}=\tilde{R}_{x}$. It is clear that the estimates (5.2), (5.3) and (5.4) also hold with $R_{x}$ instead of $\tilde{R}_{x}$. Further, it turns out that if $\mu\left(B\left(x, R_{x}\right)\right) \leq \theta_{0} R_{x}$, then

$$
\mu\left(B\left(x, \varepsilon^{-6} R_{x}\right)\right)=\mu\left(B\left(x, \tilde{R}_{x}\right)\right) \leq \theta_{0} \tilde{R}_{x}=\varepsilon^{4} R_{x} .
$$

In other words, if the average density $\mu\left(B\left(x, R_{x}\right)\right) / R_{x}$ is very small, then also the average density of the ball $B\left(x, \varepsilon^{-6} R_{x}\right)$ is small.

By a Besicovitch-Vitali type covering theorem (see [13, Theorem 2.8]), since $\mu$-a.e. $x \in E$ is the center of arbitrarily small balls of the type $B\left(x, R_{x}\right)$, there exists a countable or finite collection of disjoint closed balls $B\left(a_{i}, R_{a_{i}}\right)$, with $a_{i} \in$ $E$, which covers $\mu$-a.e. $E$. To simplify notation we will write $R_{i}:=R_{a_{i}}$ and $B_{i}:=B\left(a_{i}, R_{i}\right)$. Now we take a finite subcollection of balls $B_{i}$ such that

$$
\mu\left(\bigcup_{i \in I} B_{i}\right) \geq \mu(E)-\varepsilon .
$$

If $\mu\left(B_{i}\right) \leq \theta_{0} R_{i}$, then we say that $B_{i}$ has low density, and otherwise that it has high density. We also denote $\widehat{B}_{i}:=\varepsilon^{-5} B_{i}$.

If $B_{i}$ is a low density ball, then we let $L_{i}$ be a segment parallel to the $x$ axis, with middle point $a_{i}$, and length $\rho \mu\left(B_{i}\right)$, where $\rho$ is some constant very close to 1 , like $\rho=1-\varepsilon$, say. To choose an appropriate segment $L_{i}$ for the high density balls, we use the next lemma.

Lemma 5.5. Let $B_{i}$ be a high density ball. Then there exists some point $b_{i} \in B_{i}$ such that

$$
\begin{gathered}
\left|a_{i}-b_{i}\right| \geq \frac{\theta_{0}}{2 M} R_{i}, \\
c_{\mu \mid 10 \hat{B}_{i}}^{2}\left(b_{i}\right) \leq \varepsilon^{130}, \\
c_{\mu}^{2}\left(a_{i}, b_{i}, 10 \widehat{B}_{i}\right) \leq \frac{\varepsilon^{130}}{\mu\left(10 \hat{B}_{i}\right)} .
\end{gathered}
$$

Assuming the lemma for the moment, if $B_{i}$ is a high density ball, we denote by $\ell_{i}$ the (infinite) line through $a_{i}$ and $b_{i}$, and we let $L_{i}$ be a segment supported on $\ell_{i}$ with length $\rho \mu\left(B_{i}\right)$ and centered at $a_{i}$. We set $F=\bigcup_{i \in I} L_{i}$.

It is straightforward to check that our construction of $F$ satisfies the conditions (a) and (b) of the Lemma 5.4, with a different small constant replacing $\varepsilon$. So we 
only have to estimate the curvature of the arc length measure on $F$. Notice that each segment $L_{i}$ is contained in $\rho\left(1+\varepsilon^{10} / 2\right) B_{i}$, with $\rho=1-\varepsilon$. It easily follows that

$$
L_{i} \subset B_{i} \quad \text { and } \quad \operatorname{dist}\left(L_{i}, \partial B_{i}\right) \geq \varepsilon R_{i} / 2 \text {, }
$$

assuming $\varepsilon$ small enough. This fact will be useful in the estimates below. Then

Proof of Lemma 5.5. It follows by Tchebychev. We set $r_{i}:=\theta_{0} R_{i} /(2 M)$.

$$
\mu\left(B\left(a_{i}, r_{i}\right)\right) \leq M r_{i}=\frac{1}{2} \theta_{0} R_{i} \leq \frac{1}{2} \mu\left(B\left(a_{i}, R_{i}\right)\right),
$$

because $B_{i}$ has high density. Thus $\mu\left(B_{i} \backslash B\left(a_{i}, r_{i}\right)\right) \geq \mu\left(B_{i}\right) / 2 \geq \theta_{0} R_{i} / 2$.

Notice that

$$
\begin{aligned}
\int_{10 \hat{B}_{i}}\left[c_{\mu \mid 10 \hat{B}_{i}}^{2}(y)+\mu\left(10 \hat{B}_{i}\right) c_{\mu}^{2}\left(a_{i}, y, 10 \hat{B}_{i}\right)\right] d \mu(y) \\
=c^{2}\left(\mu_{\mid 10 \hat{B}_{i}}\right)+\mu\left(10 \hat{B}_{i}\right) c_{\mu \mid 10 \hat{B}_{i}}^{2}\left(a_{i}\right) \leq 2 \varepsilon^{150} \mu\left(10 \hat{B}_{i}\right),
\end{aligned}
$$

since (5.3) and (5.4) hold for $10 \hat{B}_{i}$, i.e., for $r=10 \varepsilon^{-5} R_{i}$. Then,

$$
\begin{aligned}
\mu\left\{y \in 10 \widehat{B}_{i} \mid\left[c_{\mu \mid 10 \hat{B}_{i}}^{2}(y)+\right.\right. & \left.\left.\mu\left(10 \widehat{B}_{i}\right) c_{\mu}^{2}\left(a_{i}, y, 10 \widehat{B}_{i}\right)\right]>\varepsilon^{130}\right\} \\
& \leq \varepsilon^{-130} 2 \varepsilon^{150} \mu\left(10 \widehat{B}_{i}\right) \leq 20 \varepsilon^{20} M \varepsilon^{-5} R_{i}=20 M \varepsilon^{15} R_{i} .
\end{aligned}
$$

If $\varepsilon$ is small enough, then $2 M \varepsilon^{15} R_{i}<\varepsilon^{10} R_{i} / 2=\theta_{0} R_{i} / 2 \leq \mu\left(B_{i} \backslash B\left(a_{i}, r_{i}\right)\right)$. Thus, there exists some point $b_{i} \in B_{i} \backslash B\left(a_{i}, r_{i}\right)$ satisfying (5.7) and (5.8).

Let us say some words about the strategy of the proof of (c) in Lemma 5.4. Although we will have to distinguish several cases, the basic idea consists in comparing the curvature of $\mathcal{H}_{\mid F}^{1}$ with the curvature of $\mu$. For instance, consider a triple of points $x, y, z \in F$, so that $x \in B_{i}, y \in B_{j}$, and $z \in B_{k}$, with $i, j, k \in I$, and take also $x^{\prime}, y^{\prime}, z^{\prime} \in \operatorname{supp}(\mu)$ such that $x^{\prime} \in B_{i}, y^{\prime} \in B_{j}$, and $z^{\prime} \in B_{k}$. Then the curvature $c(x, y, z)$ will be very close to $c\left(x^{\prime}, y^{\prime}, z^{\prime}\right)$ if the balls $B_{i}, B_{j}, B_{k}$ are very far from one another. Suppose now that the balls $B_{i}, B_{j}, B_{k}$ are close to each other and all have high density. In this case, we will not use a direct comparison argument. Instead, roughly speaking, we will show that the curvature corresponding to the triples $(x, y, z)$ with $x \in B_{i}, y \in B_{j}, z \in B_{k}$ is small because the segments $L_{i}, L_{j}, L_{k}$ are very close to a common straight line.

5.4. Preliminary estimates for the proof of Lemma 5.4. Given a ball $B_{i}$, we denote by $V_{i}$ and $\hat{V}_{i}$ the infinite strips

$$
V_{i}:=U_{\left(\varepsilon^{30} R_{i}\right)}\left(\ell_{i}\right) \quad \text { and } \quad \hat{V}_{i}:=U_{\left(\varepsilon^{5} R_{i}\right)}\left(\ell_{i}\right) .
$$


Recall that $\ell_{i}$ is the line which supports $L_{i}$. Notice that $\hat{V}_{i}$ is a strip much thicker than $V_{i}$, with the same axis $\ell_{i}$.

Lemma 5.6. If $B_{i}, i \in I$, is a high density ball, then

$$
\mu\left(B_{i} \backslash V_{i}\right) \leq 2 \varepsilon^{35} R_{i} \text { and } \mu\left(10 \hat{B}_{i} \backslash V_{i}\right) \leq \varepsilon^{24} R_{i} .
$$

Proof. By Lemma 5.5 we have

$$
c_{\mu}^{2}\left(a_{i}, b_{i}, 10 \widehat{B}_{i}\right) \leq \frac{\varepsilon^{130}}{\mu\left(10 \widehat{B}_{i}\right)} .
$$

In the proof of Lemma 3.2 we have seen that given $\delta>0, E_{0} \subset \mathbb{C}$ and $x_{0}, z_{0} \in E_{0}$ such that $c^{2}\left(x_{0}, z_{0}, E_{0}\right) \leq \delta / \mu\left(E_{0}\right)$, we have

$$
\mu\left\{y \in E_{0} \mid \operatorname{dist}(y, L)>\lambda \operatorname{diam}\left(E_{0}\right)\right\} \leq \frac{\operatorname{diam}\left(E_{0}\right) \delta^{1 / 2}}{2 \lambda},
$$

where $L$ is the line through $x_{0}, z_{0}$. If we replace $E_{0}$ by $10 \hat{B}_{i}$, and $x_{0}, z_{0}$ by $a_{i}, b_{i}$, we get

$$
\mu\left\{y \in 10 \widehat{B}_{i} \mid \operatorname{dist}\left(y, L_{i}\right)>\lambda \operatorname{diam}\left(10 \widehat{B}_{i}\right)\right\} \leq \frac{\operatorname{diam}\left(10 \widehat{B}_{i}\right) \varepsilon^{65}}{2 \lambda} .
$$

If we choose $\lambda=\varepsilon^{30} R_{i} / \operatorname{diam}\left(10 \widehat{B}_{i}\right)$, we obtain

$$
\mu\left(10 \hat{B}_{i} \backslash V_{i}\right) \leq \frac{\operatorname{diam}\left(10 \hat{B}_{i}\right)^{2} \varepsilon^{65}}{2 \varepsilon^{30} R_{i}}=\frac{400 \varepsilon^{-10} R_{i}^{2} \varepsilon^{65}}{2 \varepsilon^{30} R_{i}} \leq \varepsilon^{24} R_{i} .
$$

Analogously, by (5.10) we also have

$$
c_{\mu}^{2}\left(a_{i}, b_{i}, B_{i}\right) \leq \frac{\varepsilon^{130}}{\mu\left(B_{i}\right)} .
$$

Then it follows easily that

$$
\mu\left(B_{i} \backslash V_{i}\right) \leq \frac{\operatorname{diam}\left(B_{i}\right)^{2} \varepsilon^{65}}{2 \varepsilon^{30} R_{i}}=2 \varepsilon^{35} R_{i} .
$$

Notice that the preceding lemma and (5.5) imply that

$$
\mu\left(10 \widehat{B}_{i} \backslash V_{i}\right) \leq \varepsilon^{4} R_{i} \quad \text { for all } i \in I .
$$

Observe that this inequality holds both for low and high density balls. 
Lemma 5.7. If $B_{i}, B_{j}$ are two high density balls such that $\widehat{B}_{i} \cap \widehat{B}_{j} \neq \emptyset$ and $\varepsilon^{4} R_{i} \leq R_{j} \leq R_{i}$, then $\ell_{j} \cap B_{j}$ is contained in $\hat{V}_{i}$. In particular, $L_{j} \subset \hat{V}_{i}$.

Proof. Notice that $\hat{B}_{j} \subset 3 \hat{B}_{i}$. We will show that

$$
c_{\mu \mid B_{i}}^{2}(x)>\varepsilon^{130} \quad \text { if } x \in 3 \hat{B}_{i} \backslash U_{\left(\varepsilon^{16} R_{i}\right)}\left(\ell_{i}\right) .
$$

Assume this estimate for the moment. Since the radius of $\hat{B}_{j}$ is $\varepsilon^{-5} R_{j} \geq \varepsilon^{-5} \varepsilon^{4} R_{i}>$ $3 R_{i}$, we have $B_{i} \subset \hat{B}_{j}$, and so, $c_{\mu \mid \hat{B}_{j}}^{2}(x)>\varepsilon^{130}$. Then, by (5.3) and Lemma 5.5, $a_{j}$ and $b_{j}$ cannot coincide with $x$. That is, $a_{j}, b_{j} \in U_{\left(\varepsilon^{16} R_{i}\right)}\left(\ell_{i}\right)$. Since $\left|a_{j}-b_{j}\right| \geq \varepsilon^{-10} R_{j} / M$ by (5.6), it is not difficult to show that the diameter of $B_{j}$ which contains $a_{j}, b_{j}$ is contained in $\hat{V}_{i}$. So $L_{j} \subset \hat{V}_{i}$.

In order to prove (5.13), first we need to show the existence of two squares $P_{i}^{1}, P_{i}^{2} \subset B_{i}$ which are separated and contain enough $\mu$-measure. Let $N$ be an integer such that $20 M / \theta_{0}<N \leq 21 M / \theta_{0}$. Consider a square $Q_{i}$ concentric with $B_{i}$ with side length $2 R_{i}$, so that $B_{i} \subset Q_{i}$. Split $Q_{i}$ into $N^{2}$ squares with side length $2 R_{i} / N$ and disjoint interiors. Among these $N^{2}$ squares, let $P_{i}^{1}$ be a square such that $\mu\left(P_{i}^{1} \cap B_{i}\right)$ is maximal. Then we have $\mu\left(P_{i}^{1} \cap B_{i}\right) \geq \mu\left(B_{i}\right) / N^{2}$ and

$$
\mu\left(3 P_{i}^{1}\right) \leq 3 M \ell\left(P_{i}^{1}\right)=\frac{6 M}{N} R_{i} \leq \frac{1}{2} \mu\left(B_{i}\right) .
$$

Among the $N^{2}$ squares contained in $Q_{i} \backslash 3 P_{i}^{1}$, let $P_{i}^{2}$ be such that $\mu\left(P_{i}^{2} \cap B_{i}\right)$ is maximal. Then, for $k=1,2$, we have

$$
\mu\left(P_{i}^{k} \cap B_{i}\right) \geq \frac{\mu\left(B_{i}\right)}{2 N^{2}} \gtrsim \frac{\theta_{0}^{3}}{M^{2}} R_{i}=\frac{\varepsilon^{30}}{M^{2}} R_{i}
$$

and

$$
\operatorname{dist}\left(P_{i}^{1}, P_{i}^{2}\right) \geq \frac{\ell\left(Q_{i}\right)}{N} \geq \frac{\theta_{0}}{21 M} 2 R_{i} .
$$

Notice that, from the preceding lemma,

$$
\mu\left(P_{i}^{1} \cap B_{i} \backslash V_{i}\right) \leq \mu\left(B_{i} \backslash V_{i}\right) \leq \varepsilon^{35} R_{i} \ll \frac{1}{2} \mu\left(P_{i}^{1} \cap B_{i}\right),
$$

and analogously with $P_{i}^{2}$.

Suppose now that $x_{1} \in P_{i}^{1} \cap V_{i}$ and $x_{2} \in P_{i}^{2} \cap V_{i}$. Let $\ell_{x_{1}, x_{2}}$ be the line through $x_{1}, x_{2}$. It is not diffcult to check that the segment $\ell_{x_{1}, x_{2}} \cap B_{i}$ is contained in a strip with axis $\ell_{i}$ and width equal to

$$
\left.\frac{C R_{i}}{\operatorname{dist}\left(x_{1}, x_{2}\right)} \text { (width of } V_{i}\right)<\frac{1}{2} \varepsilon^{19} R_{i},
$$


by (5.15). Then, for any $x \in 3 \widehat{B}_{i} \backslash U_{\left(\varepsilon^{16} R_{i}\right)}\left(\ell_{i}\right)$, we have

$$
c\left(x, x_{1}, x_{2}\right)=\frac{\operatorname{dist}\left(x, L_{x_{1}, x_{2}}\right)}{\left|x-x_{1}\right|\left|x-x_{2}\right|} \gtrsim \frac{\varepsilon^{16} R_{i}}{\varepsilon^{-10} R_{i}^{2}}=\frac{\varepsilon^{26}}{R_{i}} .
$$

Thus, by (5.14),

$$
\begin{aligned}
c_{\mu \mid B_{i}}^{2}(x) & \geq \iint_{\substack{x_{1} \in P_{i}^{1} \cap V_{i} \\
x_{2} \in P_{i}^{2} \cap V_{i}}} c\left(x, x_{1}, x_{2}\right)^{2} d \mu\left(x_{1}\right) d \mu\left(x_{2}\right) \\
& \gtrsim \frac{\varepsilon^{52}}{R_{i}^{2}} \mu\left(P_{i}^{1} \cap V_{i}\right) \mu\left(P_{i}^{2} \cap V_{i}\right) \\
& \gtrsim \frac{\varepsilon^{52}}{R_{i}^{2}} M^{-4} \varepsilon^{60} R_{i}^{2}=M^{-4} \varepsilon^{112} \gg \varepsilon^{130} .
\end{aligned}
$$

5.5. Estimate of the curvature of $\mathcal{H}_{\mid F}^{1}$. Let us introduce some terminology. We denote by $F_{L D}$ and $F_{H D}$ the union of those segments $L_{i}, i \in I$, contained in balls of low and high density respectively. If $x \in \bigcup_{i \in I} B_{i}$, we denote by $B_{x}$ be the ball $B_{i}, i \in I$, which contains $x$. Analogously, $\hat{B}_{x}, a_{x}, b_{x}$, and $L_{x}$ stand for $\hat{B}_{i}$, $a_{i}, b_{i}$, and $L_{i}$ respectively. To simplify notation, we also write $\sigma:=\mathcal{H}_{\mid F}^{1}$.

By comparison with $\mu$, it is not difficult to check that $\sigma$ has linear growth with constant $\lesssim M$. That is, $\sigma(B(x, r)) \leq C M r$ for all $x, r$.

We denote

$$
\begin{aligned}
& A_{1}:=\left\{(x, y, z) \in\left(\bigcup_{i \in I} B_{i}\right)^{3} \mid B_{x}=B_{y} \neq B_{z}\right\}, \\
& A_{2}:=\left\{(x, y, z) \in\left(\bigcup_{i \in I} B_{i}\right)^{3} \mid B_{x} \neq B_{y} \neq B_{z} \neq B_{x}\right\}, \\
& A_{3}:=\left\{(x, y, z) \in A_{2} \mid \hat{B}_{x} \cap \hat{B}_{y} \neq \emptyset, \hat{B}_{x} \cap \hat{B}_{z} \neq \emptyset, \hat{B}_{y} \cap \hat{B}_{z} \neq \emptyset\right\}, \\
& A_{4}:=\left\{(x, y, z) \in A_{2} \mid \hat{B}_{x}, \hat{B}_{y}, \hat{B}_{z} \text { pairwise disjoint }\right\},
\end{aligned}
$$

and also

$$
\begin{aligned}
& A_{5}^{x}:=\left\{(x, y, z) \in A_{2} \mid \hat{B}_{x} \cap \hat{B}_{y}=\emptyset, \hat{B}_{x} \cap \hat{B}_{z}=\emptyset, \hat{B}_{y} \cap \hat{B}_{z} \neq \emptyset\right\}, \\
& A_{6}^{x}:=\left\{(x, y, z) \in A_{2} \mid \hat{B}_{x} \cap \hat{B}_{y} \neq \emptyset, \hat{B}_{x} \cap \hat{B}_{z} \neq \emptyset, \hat{B}_{y} \cap \hat{B}_{z}=\emptyset\right\} .
\end{aligned}
$$

Interchanging, $x$ by $y$ and $z$, we define analogously $A_{5}^{y}, A_{5}^{z}, A_{6}^{y}$ and $A_{6}^{z}$. Observe that the sets $A_{1}, \ldots, A_{6}^{z}$ are pairwise disjoint. 
Since $c^{2}\left(\sigma_{\mid L_{i}}\right)=0$ for all $i$, we have

$$
\begin{aligned}
& c^{2}(\sigma)=\iiint_{\left(\cup_{i \in I} B_{i}\right)^{3}} c(x, y, z)^{2} d \sigma(x) d \sigma(y) d \sigma(z) \\
= & 3 \iiint_{A_{1}} \cdots+\iiint_{A_{2}} \cdots \\
= & 3 \iiint_{A_{1}} \cdots+\iiint_{A_{3}} \cdots+\iiint_{A_{4}} \cdots+\iiint_{A_{5}^{x} \cup A_{5}^{y} \cup A_{5}^{z}} \cdots+\iiint_{A_{6}^{x} \cup A_{6}^{y} \cup A_{6}^{z}} \cdots \\
= & 3 I_{1}+I_{3}+I_{4}+I_{5}+I_{6} .
\end{aligned}
$$

- Estimates for $I_{4}$. In this case, since the balls $\hat{B}_{x}, \hat{B}_{y}$ and $\hat{B}_{z}$ are pairwise disjoint, the segments $L_{x}, L_{y}$ and $L_{z}$ are very far from one another, and so if $x^{\prime} \in B_{x}, y^{\prime} \in B_{y}$ and $z^{\prime} \in B_{z}$, by Lemma 5.2 we have

$$
\begin{aligned}
& c(x, y, z) \leq c\left(x^{\prime}, y^{\prime}, z^{\prime}\right)+\frac{C R_{x}}{|x-y||x-z|} \\
& +\frac{C R_{y}}{|y-x||y-z|}+\frac{C R_{z}}{|z-x||z-y|} \\
& \quad=: c\left(x^{\prime}, y^{\prime}, z^{\prime}\right)+C\left[T_{x}(x, y, z)+T_{y}(x, y, z)+T_{z}(x, y, z)\right] .
\end{aligned}
$$

Squaring this inequality it easily follows that

$$
\begin{aligned}
c(x, y, z)^{2} \leq(1 & +\varepsilon) \mathcal{c}\left(x^{\prime}, y^{\prime}, z^{\prime}\right)^{2} \\
& +C \varepsilon^{-1}\left[T_{x}(x, y, z)^{2}+T_{y}(x, y, z)^{2}+T_{z}(x, y, z)^{2}\right] .
\end{aligned}
$$

Let $B_{i}, B_{j}, B_{k}$, with $i, j, k \in I$, be balls far from each other as in the definition of $A_{4}$. From the preceding estimate, integrating with respect to $\mu$ and taking into account that $\mu\left(B_{h}\right)=\sigma\left(B_{h}\right)$ for all $h \in I$ and that $T_{x}(x, y, z) \approx T_{x}\left(x^{\prime}, y^{\prime}, z^{\prime}\right)$ and analogously for $T_{y}$ and $T_{z}$, we get

$$
\begin{aligned}
& c(x, y, z)^{2} \sigma\left(B_{i}\right) \sigma\left(B_{j}\right) \sigma\left(B_{k}\right) \leq \\
& \leq(1+\varepsilon) c_{\mu}^{2}\left(B_{i}, B_{j}, B_{k}\right)+C \varepsilon^{-1} \iiint_{\substack{x \in B_{i} \\
y \in B_{j}}}\left[T_{x}(x, y, z)^{2}+T_{y}(x, y, z)^{2}\right. \\
&+
\end{aligned}
$$

for all $x \in B_{i}, y \in B_{j}, z \in B_{k}$. If we take means with respect to $\sigma$ for $x \in B_{i}$, $y \in B_{j}, z \in B_{k}$, and then we sum over the balls $B_{i}, B_{j}, B_{k}$ in the definition of $A_{4}$, 
we obtain

$$
\begin{aligned}
& I_{4} \leq(1+\varepsilon) \iiint_{(x, y, z) \in A_{4}} c(x, y, z)^{2} d \mu(x) d \mu(y) d \mu(z) \\
&+C \varepsilon^{-1} \iiint_{(x, y, z) \in A_{4}}[ T_{x}(x, y, z)^{2}+T_{y}(x, y, z)^{2} \\
&\left.+T_{z}(x, y, z)^{2}\right] d \mu(x) d \mu(y) d \mu(z) .
\end{aligned}
$$

Let us estimate the last integral in (5.18). We have

$$
\begin{aligned}
& \iiint_{(x, y, z) \in A_{4}} T_{x}(x, y, z)^{2} d \mu(x) d \mu(y) d \mu(z) \leq \\
& \quad \leq \iiint_{\substack{|x-y|>\varepsilon^{-5} R_{x} / 2 \\
|x-z|>\varepsilon^{-5} R_{x} / 2}} \cdots \\
& \quad=\int\left(\int_{|x-y|>\varepsilon^{-5} R_{x} / 2} \frac{R_{x}}{|x-y|^{2}} d \mu(y)\right)^{2} d \mu(x) \\
& \quad \lesssim M^{2} \varepsilon^{10} \mu(E) .
\end{aligned}
$$

We have analogous estimates for the integrals of $T_{y}(\cdots)^{2}$ and $T_{z}(\cdots)^{2}$. Thus,

$$
I_{4} \leq(1+\varepsilon) \iiint_{(x, y, z) \in A_{4}} c(x, y, z)^{2} d \mu(x) d \mu(y) d \mu(z)+C M^{2} \varepsilon^{9} \mu(E) .
$$

- Estimates for $I_{5}$. We have

$$
I_{5}=\iiint_{A_{5}^{x}} \cdots+\iiint_{A_{5}^{y}} \cdots+\iiint_{A_{5}^{z}} \cdots=: I_{5}^{x}+I_{5}^{y}+I_{5}^{z} .
$$

Let us consider the integral $I_{5}^{x}$. Given $(x, y, z) \in A_{5}^{x}$, consider $x^{\prime} \in B_{x}, y^{\prime} \in B_{y}$ and $z^{\prime} \in B_{z}$. It is easy to check that the same estimates (5.17) and (5.18) used for $I_{4}$ hold in this case. Notice that in the domain of integration of $I_{5}^{x}$ we have

$$
|x-y| \geq \varepsilon^{-5} \frac{R_{x}}{2} \quad \text { and } \quad|x-z| \geq \varepsilon^{-5} \frac{R_{x}}{2} .
$$

Thus, (5.19) is also valid for $I_{5}^{x}$. However, the analogous inequalities for $T_{y}$ and $T_{z}$ fail. Nevertheless, some easy modifications are enough to deal with $T_{y}$ and $T_{z}$. Regarding $T_{y}$ we have 


$$
\begin{aligned}
& \iiint_{(x, y, z) \in A_{5}^{x}} T_{y}(x, y, z)^{2} d \mu(x) d \mu(y) d \mu(z) \\
\leq & \iiint_{|y-x|>\varepsilon^{-5} R_{y} / 2} \cdots \\
= & \int\left(\int_{|y-z|>\varepsilon R_{y} / 2}\right. \\
\leqslant & M^{2} \varepsilon^{5} \varepsilon^{-1} \mu(E)=M^{2} \varepsilon^{4} \mu(E) .
\end{aligned}
$$

The term $T_{z}$ is estimated similarly, and then we get

$$
I_{5}^{x} \leq(1+\varepsilon) \iiint_{(x, y, z) \in A_{5}^{x}} c(x, y, z)^{2} d \mu(x) d \mu(y) d \mu(z)+C M^{2} \varepsilon^{3} \mu(E) .
$$

Analogous inequalities hold for $I_{5}^{y}$ and $I_{5}^{z}$. So we have

$$
\begin{aligned}
I_{5} \leq(1+\varepsilon) \iiint_{(x, y, z) \in A_{5}^{x} \cup A_{5}^{y} \cup A_{5}^{z}} c(x, y, z)^{2} d \mu(x) d \mu(y) d \mu(z) & \\
& +C M^{2} \varepsilon^{3} \mu(E) .
\end{aligned}
$$

- Estimates for $I_{6}$. We split $I_{6}$ as follows:

$$
I_{6}=\iiint_{A_{6}^{x}} \cdots+\iiint_{A_{6}^{y}} \cdots+\iiint_{A_{6}^{z}} \cdots=: I_{6}^{x}+I_{6}^{y}+I_{6}^{z} .
$$

Let us consider the integral $I_{6}^{x}$. Given $(x, y, z) \in A_{6}^{x}$, consider $y^{\prime} \in B_{y}$ and $z^{\prime} \in B_{z}$. Using Lemma 5.2 it is easy to check that

$$
\begin{aligned}
c(x, y, z) & \leq c\left(x, y^{\prime}, z^{\prime}\right)+\frac{C R_{y}}{|y-x||y-z|}+\frac{C R_{z}}{|z-x||z-y|} \\
& =: c\left(x, y^{\prime}, z^{\prime}\right)+C\left[T_{y}(x, y, z)+T_{z}(x, y, z)\right] .
\end{aligned}
$$

Operating as in the case of $I_{4}$, it follows that

$$
\begin{aligned}
I_{6}^{x} \leq(1+\varepsilon) & \iiint_{(x, y, z) \in A_{6}^{x}} c(x, y, z)^{2} d \sigma(x) d \mu(y) d \mu(z) \\
& +C \varepsilon^{-1} \iiint_{(x, y, z) \in A_{6}^{x}}\left[T_{y}(x, y, z)^{2}+T_{z}(x, y, z)^{2}\right] d \sigma(x) d \mu(y) d \mu(z) .
\end{aligned}
$$


Observe that if $(x, y, z) \in A_{6}^{x}$, then $|y-z| \geq \varepsilon^{-5}\left(R_{y}+R_{z}\right) / 2$. As a consequence, the terms $T_{y}$ and $T_{z}$ can be estimated similarly to the case of $I_{5}^{x}$ :

$$
\begin{aligned}
& \iiint_{(x, y, z) \in A_{G}^{x}} T_{y}(x, y, z)^{2} d \sigma(x) d \mu(y) d \mu(z) \leq \\
\leq & \iiint_{|y-x|>\varepsilon R_{y} / 2} \cdots \\
= & \int\left(\int_{|y-z|>\varepsilon^{-5} R_{y} / 2}\right. \\
\lesssim & M^{2} \varepsilon^{-1} \varepsilon^{5} \mu(E)=M^{2} \varepsilon^{4} \mu(E) .
\end{aligned}
$$

We have an analogous estimate for the term $T_{z}$. Then we obtain

$$
I_{6}^{x} \leq(1+\varepsilon) \iiint_{(x, y, z) \in A_{6}^{x}} c(x, y, z)^{2} d \sigma(x) d \mu(y) d \mu(z)+C M^{2} \varepsilon^{3} \mu(E) .
$$

To estimate the triple integral above we set

$$
\begin{aligned}
& \iiint_{(x, y, z) \in A_{6}^{x}} c(x, y, z)^{2} d \sigma(x) d \mu(y) d \mu(z)= \\
& =\iiint_{\substack{(x, y, z) \in A_{6}^{x} \\
y \notin \hat{B}_{x} \text { or } z \notin \hat{B}_{x}}} \cdots+\iiint_{\substack{(x, y, z) \in A_{6}^{x} \\
y, z \in \hat{B}_{x}}} \cdots=: J_{1}+J_{2} .
\end{aligned}
$$

Taking into account that in the domain of integration of $J_{1}$ either $y$ or $z$ is very far from $B_{x}$, operating as above it is easy to check that

$$
J_{1} \leq(1+\varepsilon) \iiint_{(x, y, z) \in A_{6}^{x}} c(x, y, z)^{2} d \mu(x) d \mu(y) d \mu(z)+C M^{2} \varepsilon^{3} \mu(E) .
$$

We leave the details for the reader.

Let us deal with $J_{2}$ now. Suppose first that $x \in F_{H D}$. That is, $B_{x}$ has high density. We intend to apply Lemma 5.3. To this end, given $x \in \operatorname{supp}(\sigma)$, consider the points $a_{x}, b_{x} \in L_{x}$, so that $x, a_{x}, b_{x}$ are collinear. Then we have $\left|x-a_{x}\right| \leq 2 R_{x} \lesssim M \varepsilon^{-10}\left|a_{x}-b_{x}\right|$ (recall Lemma 5.5), and for $y, z \in \hat{B}_{x} \backslash B_{x}$, it is easy to check that $\left|y-a_{x}\right| \lesssim \varepsilon^{-1}|y-x|$ and $\left|z-a_{x}\right| \lesssim \varepsilon^{-1}|z-x|$, and similarly with $b_{x}$ instead of $a_{x}$. Then we obtain

$$
c(x, y, z) \lesssim M \varepsilon^{-12}\left(c\left(a_{x}, y, z\right)+c\left(b_{x}, y, z\right)\right),
$$


by Lemma 5.3. If we square and integrate this inequality over $y, z \in \hat{B}_{x} \backslash B_{x}$ with respect to $\mu$, we get

$$
\begin{aligned}
& \iint_{y, z \in \hat{B}_{x} \backslash B_{x}} c(x, y, z)^{2} d \mu(y) d \mu(z) \lesssim \\
& \quad \lesssim M^{2} \varepsilon^{-24}\left(c_{\mu \mid \hat{B}_{x}}^{2}\left(a_{x}\right)+c_{\mu \mid \hat{B}_{x}}^{2}\left(b_{x}\right)\right) \lesssim M^{2} \varepsilon^{106},
\end{aligned}
$$

since $c_{\mu \mid \hat{B}_{x}}^{2}\left(a_{x}\right), c_{\mu \mid \hat{B}_{x}}^{2}\left(b_{x}\right) \leq \varepsilon^{130}$, by Lemma 5.5. Suppose now that $x \in F_{L D}$. In this case, we use the fact that $\mu\left(\hat{B}_{x}\right)$ is very small, and then we obtain

$$
\begin{aligned}
& \iint_{y, z \in \hat{B}_{x} \backslash B_{x}} c(x, y, z)^{2} d \mu(y) d \mu(z) \lesssim \\
& \quad \lesssim \iint_{\varepsilon R_{x} / 2 \leq|y-x| \leq \varepsilon^{-5} R_{x}} \frac{1}{|x-z|^{2}} d \mu(y) d \mu(z) \\
& \quad \lesssim \int_{\varepsilon R_{x} / 2 \leq|y-x| \leq \varepsilon^{-5} R_{x}} \frac{M}{|x-y|} d \mu(y) \\
& \quad \lesssim \frac{M \mu\left(\hat{B}_{x}\right)}{\varepsilon R_{x}} \lesssim \frac{M \varepsilon^{4} R_{x}}{\varepsilon R_{x}}=M \varepsilon^{3} .
\end{aligned}
$$

By (5.20) and (5.21), integrating over $x \in F$ with respect to $\sigma$, we get

$$
\begin{gathered}
\iiint_{\substack{(x, y, z) \in A_{6}^{x} \\
y, z \in \hat{B}_{x}}} c(x, y, z)^{2} d \sigma(x) d \mu(y) d \mu(z) \\
\quad \lesssim\left(M^{2} \varepsilon^{106}+M \varepsilon^{3}\right) \sigma(F) \lesssim M^{2} \varepsilon^{3} \mu(E) .
\end{gathered}
$$

Therefore,

$$
I_{6}^{x} \leq(1+\varepsilon)^{2} \iiint_{(x, y, z) \in A_{6}^{x}} c(x, y, z)^{2} d \mu(x) d \mu(y) d \mu(z)+C M^{2} \varepsilon^{3} \mu(E) .
$$

Analogous estimates hold for $I_{6}^{y}$ and $I_{6}^{z}$.

- Estimates for $I_{1}$. We set

$$
I_{1}=\iiint_{\substack{(x, y, z) \in A_{1} \\|x-z|>\varepsilon^{-1} R_{x}}} \cdots+\iiint_{\substack{(x, y, z) \in A_{1} \\|x-z| \leq \varepsilon^{-1} R_{x}}} \cdots=: I_{1,1}+I_{1,2} .
$$

First we deal with $I_{1,1}$ :

$$
\begin{aligned}
I_{1,1} & \lesssim \iint_{\substack{x \in F \\
y \in L_{x}}}\left(\int_{|x-z|>\varepsilon^{-1} R_{x}} \frac{1}{|x-z|^{2}} d \sigma(z)\right) d \sigma(x) d \sigma(y) \\
& \lesssim \int_{x \in F} \sigma\left(L_{x}\right) \frac{M}{\varepsilon^{-1} R_{x}} d \sigma(x)=M \varepsilon \sigma(F) \leq M \varepsilon \mu(E) .
\end{aligned}
$$


To estimate $I_{1,2}$ we split the integral as follows:

$$
\begin{aligned}
& I_{1,2} \leq \iiint_{\substack{(x, y, z) \in A_{1} \\
x \in F_{L D} \\
|x-z| \leq \varepsilon^{-1} R_{x}}} \cdots+\iiint_{\substack{x \in F_{H D} \\
|x-z| \leq \varepsilon^{-1} R_{x} \\
R_{z} \leq R_{x} \\
B_{z} \cap V_{x}=\emptyset}} \cdots+\iiint_{\substack{x \in F_{H D} \\
|x-z| \leq \varepsilon^{-1} R_{x} \\
R_{z} \leq R_{x} \\
B_{z} \cap V_{x} \neq \emptyset}} \cdots \\
& +\iiint_{\substack{(x, y, z) \in A_{1} \\
\left|x \in F_{H D}\\
\right| x-\mid \leq \varepsilon^{-1} R_{x} \\
R_{z}>R_{x} \\
z \in F_{L D}}} \cdots+\iiint_{\substack{(x, y, z) \in A_{1} \\
\mid x F_{H D} \\
R_{z}>R_{x} \\
z \in \varepsilon_{H}^{-1}}} \cdots=: S_{1}+S_{2}+S_{3}+S_{4}+S_{5} .
\end{aligned}
$$

To estimate $S_{1}$ we will use that $|x-z| \geq \varepsilon R_{x} / 2$ and that $\mu\left(\hat{B}_{x}\right)$ is small:

$$
\begin{aligned}
& S_{1} \lesssim \iint_{\substack{x \in F_{L D} \\
\varepsilon R_{x} / 2 \leq|x-z| \leq \varepsilon^{-1} R_{x} \\
z \notin B_{x}}} \frac{\sigma\left(L_{x}\right)}{|x-z|^{2}} d \sigma(x) d \sigma(z) \\
& \lesssim \int_{x \in F_{L D}} \frac{R_{x}}{\varepsilon^{2} R_{x}^{2}} \sigma\left(B\left(x, \varepsilon^{-1} R_{x}\right) \backslash B_{x}\right) d \sigma(x) .
\end{aligned}
$$

Notice now that for $x \in \operatorname{supp}(\sigma)$ and $z \in \operatorname{supp}(\sigma) \cap B\left(x, \varepsilon^{-1} R_{x}\right) \backslash B_{x}$ we have

$$
|x-z| \geq \varepsilon\left(R_{x}+R_{z}\right) / 2 .
$$

This implies that $R_{z} \leq C \varepsilon^{-2} R_{x}$, and so $B_{z} \subset B\left(x, C \varepsilon^{-2} R_{x}\right)$. Thus

$$
\sigma\left(B\left(x, \varepsilon^{-1} R_{x}\right) \backslash B_{x}\right) \leq \mu\left(B\left(x, C \varepsilon^{-2} R_{x}\right)\right) .
$$

Since $B_{x}$ has low density, $\mu\left(B\left(x, C \varepsilon^{-2} R_{x}\right)\right) \leq \mu\left(\hat{B}_{x}\right) \leq \varepsilon^{4} R_{x}$. Therefore,

$$
S_{1} \lesssim \varepsilon^{2} \sigma(F) \leq C \varepsilon^{2} \mu(E) .
$$

For $S_{2}$ we take into account that $c(x, y, z) \lesssim|x-z|^{-1} \lesssim \varepsilon^{-1} R_{x}^{-1}$ and that $\mu\left(\hat{B}_{x} \backslash V_{x}\right)$ is very small, by Lemma 5.6 :

$$
S_{2} \lesssim \int_{x \in F_{H D}} \frac{\sigma\left(L_{x}\right)}{\varepsilon^{2} R_{x}^{2}} \mu\left(\hat{B}_{x} \backslash V_{x}\right) d \sigma(x) \lesssim \varepsilon^{22} \sigma(F) \leq \varepsilon^{22} \mu(E) .
$$

Let us consider $S_{3}$ now. Consider $(x, y, z)$ in the domain of integration of $S_{3}$. If $R_{z} \geq \varepsilon^{4} R_{x}$, then $L_{z} \subset \hat{V}_{x}$ by Lemma 5.7. If $R_{z}<\varepsilon^{4} R_{x}$ and $B_{z} \cap V_{x} \neq \emptyset$, then $z \in U_{3 \varepsilon^{4} R_{x}}\left(\ell_{x}\right)$. So in any case

$$
\operatorname{dist}\left(z, \ell_{x}\right) \lesssim \varepsilon^{4} R_{x} .
$$


Thus,

$$
c(x, y, z) \lesssim \frac{\varepsilon^{4} R_{x}}{|x-z||y-z|} \lesssim \frac{\varepsilon^{4} R_{x}}{\varepsilon^{2} R_{x}^{2}}=\frac{\varepsilon^{2}}{R_{x}} .
$$

So, using (5.22) again, we obtain

$$
S_{3} \lesssim \int_{x \in F} \frac{\sigma\left(L_{x}\right) \varepsilon^{4} \mu\left(B\left(x, C \varepsilon^{-2} R_{x}\right)\right)}{R_{x}^{2}} d \sigma(x) \lesssim M \varepsilon^{2} \sigma(F) \lesssim M \varepsilon^{2} \mu(F) .
$$

For $S_{4}$, observe that $\hat{B}_{x} \subset 3 \widehat{B}_{z}$ and since $B_{z}$ is a low density ball, $\mu\left(\hat{B}_{x}\right) \leq$ $\mu\left(3 \hat{B}_{z}\right) \leq \varepsilon^{4} R_{z}$. Then,

$$
c(x, y, z)^{2} \lesssim \frac{1}{|x-z|^{2}} \lesssim \frac{1}{\varepsilon^{2} R_{x} R_{z}} \lesssim \frac{\varepsilon^{2}}{R_{x} \mu\left(\hat{B}_{x}\right)} .
$$

Therefore, using (5.22),

$$
\begin{aligned}
S_{4} & \lesssim \int_{x \in F} \frac{\sigma\left(L_{x}\right) \varepsilon^{2} \mu\left(B\left(x, C \varepsilon^{-2} R_{x}\right)\right)}{R_{x} \mu\left(\hat{B}_{x}\right)} d \sigma(x) \\
& \lesssim \int_{x \in F} \frac{\varepsilon^{2} \mu\left(\hat{B}_{x}\right)}{\mu\left(\hat{B}_{x}\right)} d \sigma(x) \leq \varepsilon^{2} \mu(E) .
\end{aligned}
$$

Finally we deal with $S_{5}$. In this case we have $C^{-1} \varepsilon^{2} R_{z} \leq R_{x} \leq R_{z}$, and $\hat{B}_{x} \subset 3 \hat{B}_{z}$. Since $B_{z}$ is a high density ball, by Lemma 5.7, $\ell_{x} \cap B_{x} \subset \hat{V}_{z}$. Since the width of $\hat{V}_{z}$ is $\varepsilon^{5} R_{z}$, we derive

$$
\sin \Varangle\left(\ell_{x}, \ell_{z}\right) \lesssim \frac{\varepsilon^{5} R_{z}}{R_{x}} \lesssim \varepsilon^{3} .
$$

Since $y, z \in \hat{V}_{z}$ and $|y-z| \geq \varepsilon R_{z} / 2$, we also have $\sin \Varangle\left(\ell_{z}, \ell_{y, z}\right) \lesssim \varepsilon^{5} R_{z} /\left(\varepsilon R_{z}\right)=$ $\varepsilon^{4}$, where $\ell_{y, z}$ stands for the line through $y$ and $z$. Thus,

$$
\sin \Varangle(x, y, z) \lesssim \sin \Varangle\left(\ell_{x}, \ell_{z}\right)+\sin \Varangle\left(\ell_{z}, \ell_{y, z}\right) \lesssim \varepsilon^{3} .
$$

So, $c(x, y, z) \lessgtr \varepsilon^{3} /|x-z|$, and we get

$$
\begin{aligned}
S_{5} & \lesssim \iint_{\substack{x \in F \\
|z-x|>\varepsilon R_{x} / 2}} \frac{\sigma\left(L_{x}\right) \varepsilon^{6}}{|x-z|^{2}} d \sigma(z) d \sigma(x) \\
& \lessgtr \int_{x \in F} \frac{M \sigma\left(L_{x}\right) \varepsilon^{6}}{\varepsilon R_{x}} d \sigma(x) \lesssim M \varepsilon^{5} \mu(E) .
\end{aligned}
$$

So we have shown that $I_{1} \lesssim M \varepsilon \mu(E)$. 
- Estimates for $I_{3}$. We denote $A_{3}^{\prime}=\left\{(x, y, z) \in A_{3} \mid R_{x} \geq R_{y}, R_{z}\right\}$. Then we have

$$
I_{3}=\iiint_{(x, y, z) \in A_{3}} \cdots \leq 3 \iiint_{A_{3}^{\prime}} \cdots .
$$

Notice that if $(x, y, z) \in A_{3}^{\prime}$, then $B_{y}, B_{z} \subset 3 \hat{B}_{x}$. We have

$$
\begin{aligned}
I_{3} & \lesssim \iiint_{\substack{(x, y, z) \in A_{3}^{\prime} \\
B_{y} \cap V_{x}=\emptyset}} \cdots+\iiint_{\substack{(x, y, z) \in A_{3}^{\prime} \\
B_{z} \cap V_{x}=\emptyset}} \cdots+\iiint_{\substack{B_{y} \cap V_{x} \neq \emptyset \\
B_{z} \cap V_{x} \neq \emptyset}}\left(x, y, A_{3}^{\prime}\right. \\
& =: I_{3,1}+I_{3,2}+I_{3,3} .
\end{aligned}
$$

To estimate $I_{3,1}$ we take into account that $\mu\left(10 \hat{B}_{x} \backslash V_{x}\right) \leq \varepsilon^{4} R_{x}$. Then we obtain

$$
\begin{aligned}
I_{3,1} & \lessgtr \iint_{\substack{x \in F \\
|x-z|>\varepsilon R_{x}}} \frac{\mu\left(3 \hat{B}_{x} \backslash V_{x}\right)}{|x-z|^{2}} d \sigma(x) d \sigma(z) \\
& \lessgtr \int_{x \in F} \frac{M \varepsilon^{4} R_{x}}{\varepsilon R_{x}} d \sigma(x) \lesssim M \varepsilon^{3} \mu(E) .
\end{aligned}
$$

Analogously, we have

$$
I_{3,2} \lesssim M \varepsilon^{3} \mu(E) .
$$

We deal with $I_{3,3}$ now. We set

$$
\begin{aligned}
& I_{3,3} \leq 2 \iiint_{B_{y} \cap V_{x} \neq \emptyset}(x, y, z) \in A_{3}^{\prime} \quad \cdots \\
& B_{z} \cap V_{x} \neq \emptyset \\
& \lesssim \iiint_{\substack{(x, y, z) \in A_{3}^{\prime} \\
|x-z| \geq|-y|}} \cdots+\iiint_{\substack{(x, y, z) \in A_{3}^{\prime} \\
|y-z| \leq \varepsilon^{2} R_{x}}} \cdots=: P_{1}+P_{2} .
\end{aligned}
$$

To estimate $P_{1}$ we use that $\sigma\left(B\left(y, \varepsilon^{2} R_{x}\right)\right) \lesssim M \varepsilon^{2} R_{x}$ :

$$
\begin{aligned}
P_{1} & \lesssim \iint_{\substack{x \in F \\
|x-y| \geq \varepsilon R_{x} / 2}} \frac{\sigma\left(B\left(y, \varepsilon^{2} R_{x}\right)\right)}{|x-y|^{2}} d \sigma(x) d \sigma(y) \\
& \lesssim \int_{x \in F} \frac{M^{2} \varepsilon^{2} R_{x}}{\varepsilon R_{x}} d \sigma(x) \lesssim M^{2} \varepsilon \mu(E) .
\end{aligned}
$$

Let us turn our attention to $P_{2}$. As in (5.23), by Lemma 5.7 it follows easily that

$$
y, z \in U_{\left(C \varepsilon^{4} R_{x}\right)}\left(\ell_{x}\right) .
$$


That is, $y, z$ lie in a very narrow strip with axis $\ell_{x}$. On the other hand, since

$$
|x-y| \geq \varepsilon \frac{R_{x}}{2}, \quad|x-y| \geq \varepsilon \frac{R_{x}}{2}, \quad \text { and } \quad|y-z|>\varepsilon^{2} R_{x},
$$

by (5.24), it is easy to see that $\sin \Varangle(x, y, z) \lesssim \varepsilon^{4} R_{x} / \varepsilon^{2} R_{x}=\varepsilon^{2}$. Thus, $c(x, y, z) \lesssim$ $\varepsilon^{2} /|x-z|$. As a consequence,

$$
\begin{aligned}
P_{2} & \lessgtr \iiint_{\substack{x \in F_{y \in B_{x} \backslash B_{x}} \\
|x-z| \geq|x-y|}} \frac{\varepsilon^{4}}{|x-z|^{2}} d \sigma(x) d \sigma(y) d \sigma(z) \\
& \lessgtr \iint_{\substack{x \in F \\
\varepsilon R_{x} / 2 \leq|x-y| \leq 3 \varepsilon^{-5} R_{x}}} \frac{M \varepsilon^{4}}{|x-y|} d \sigma(x) d \sigma(y) \\
& \lessgtr M^{2} \varepsilon^{4}\left(\log \frac{\varepsilon^{-5} R_{x}}{\varepsilon R_{x}}\right) \mu(E) \lesssim M^{2} \varepsilon^{3} \mu(E) .
\end{aligned}
$$

So we have shown that $I_{3} \lesssim M^{2} \varepsilon \mu(E)$.

- End of the proof of Lemma 5.4. By the estimates obtained for $I_{1}, \ldots, I_{5}$, we get

$$
c^{2}\left(\mathcal{H}_{\mid F}^{1}\right)=c^{2}(\sigma) \leq(1+\varepsilon)^{2} c^{2}(\mu)+C M^{2} \varepsilon \mu(E) .
$$

Acknowledgement. This research was partially supported by grants MTM200400519 and Acción Integrada HF2004-0208 (Spain), and 2001-SGR-00431 (Generalitat de Catalunya).

\section{REFERENCES}

[1] Lars V. Ahlfors, Bounded analytic functions, Duke Math. J. 14 (1947), 1-11. MR0021108 $(9,24 a)$

[2] GUY DAVID, Unrectifiable 1-sets have vanishing analytic capacity, Rev. Mat. Iberoamericana 14 (1998), 369-479. MR1654535 (99i:42018) (English, with English and French summaries)

[3] GUY DAVID and S. SEMmes, Singular Integrals and Rectifiable Sets in $\mathbf{R}^{n}$ : Beyond Lipschitz Graphs, Astérisque, vol. 193, 1991. MR1113517 (92j:42016) (English, with French summary)

[4] K. J. FalCONER, The Geometry of Fractal Sets, Cambridge Tracts in Mathematics, vol. 85, Cambridge University Press, Cambridge, 1986, ISBN 0-521-25694-1, 0-521-33705-4. MR867284 (88d:28001)

[5] Theodore W. Gamelin, Uniform Algebras, Prentice-Hall Inc., Englewood Cliffs, N. J., 1969. MR0410387 (53\#14137)

[6] Paul R. Garabedian, The classes $L_{p}$ and conformal mapping, Trans. Amer. Math. Soc. 69 (1950), 392-415. MR0039072 (12,492a)

[7] John Garnett, Analytic Capacity and Measure, Springer-Verlag, Berlin, 1972. MR0454006 (56 \#12257)

[8] Peter W. Jones, Rectifiable sets and the traveling salesman problem, Invent. Math. 102 (1990), 1-15, http://dx.doi.org/10.1007/BF01233418. MR1069238 (91i:26016) 
[9] Peter W. Jones and Takafumi Murai, Positive analytic capacity but zero Buffon needle probability, Pacific J. Math. 133 (1988), 99-114. MR936358 (89m:30050)

[10] JEAn-Christophe LÉGER, Menger curvature and rectifiability, Ann. of Math. (2) 149 (1999), 831-869. MR1709304 (2001c:49069)

[11] GILAD LERMAN, Quantifying curvelike structures of measures by using $L^{2}$ Jones quantities, Comm. Pure Appl. Math. 56 (2003), 1294-1365, http://dx.doi.org/10.1002/cpa.10096. MR1980856 (2004c:42035)

[12] PertTi MatTila, Smooth maps, null-sets for integralgeometric measure and analytic capacity, Ann. of Math. (2) 123 (1986), 303-309. MR835764 (87d:28010)

[13] _ Geometry of Sets and Measures in Euclidean Spaces, Cambridge Studies in Advanced Mathematics, vol. 44, Cambridge University Press, Cambridge, 1995, ISBN 0-521-46576-1, 0-521-65595-1. MR1333890 (96h:28006)

[14] - Hausdorff dimension, projections, and the Fourier transform, Publ. Mat. 48 (2004), 3-48. MR2044636 (2004k:28018)

[15] Pertti Mattila, Mark S. Melnikov, and Joan Verdera, The Cauchy integral, analytic capacity, and uniform rectifiability, Ann. of Math. (2) 144 (1996), 127-136. MR1405945 (97k:31004)

[16] Mark S. Melnikov, Analytic capacity: a discrete approach and the curvature of measure, Sbornik: Mathematics 186 (1995), 827-846, http://dx.doi.org/10.1070/SM1995v186n06ABEH000045. MR1349014 (96f:30020)

[17] Mark S. Melnikov and Joan Verdera, A geometric proof of the $L^{2}$ boundedness of the Cauchy integral on Lipschitz graphs, Internat. Math. Res. Notices (1995), 325-331, http://dx.doi.org/10.1155/S1073792895000249. MR1350687 (96f:45011)

[18] Hervé Pajot, Analytic Capacity, Rectifiability, Menger Curvature and the Cauchy Integral, Lecture Notes in Mathematics, vol. 1799, Springer-Verlag, Berlin, 2002, ISBN 3-540-00001-1. MR1952175 (2004d:28009)

[19] XAVIER TOLSA, L2-boundedness of the Cauchy integral operator for continuous measures, Duke Math. J. 98 (1999), 269-304, http://dx.doi.org/10.1215/S0012-7094-99-09808-3. MR1695200 (2000d:31001)

[20] On the analytic capacity $\gamma_{+}$, Indiana Univ. Math. J. 51 (2002), 317-343, http://dx.doi.org/10.1512/iumj.2002.51.2202. MR1909292 (2003m:31004)

[21] _ Painlevés problem and the semiadditivity of analytic capacity, Acta Math. 190 (2003), 105-149. MR1982794 (2005c:30020)

[22] $L^{2}$ boundedness of the Cauchy transform implies $L^{2}$ boundedness of all Calderón-Zygmund operators associated to odd kernels, Publ. Mat. 48 (2004), 445-479. MR2091015 (2005e:42053)

Institució Catalana de Recerca i Estudis Avançats (ICREA)

and Departament de Matemàtiques

Universitat Autònoma de Barcelona

Barcelona, Spain.

E-MAIL: xtolsa@mat.uab.es

KEY WORDS AND PHRASES: curvature of measures, rectifiability, analytic capacity. 2000 Mathematics Subject Classification: 30C85, 28 A75.

Received: February 1, 2005. 